\title{
The Gaia-ESO Survey: radial distribution of abundances in the Galactic disc from open clusters and young-field stars ${ }^{\star}$
}

\author{
L. Magrini ${ }^{1}$, S. Randich ${ }^{1}$, G. Kordopatis ${ }^{2}$, N. Prantzos $^{3}$, D. Romano ${ }^{4}$, A. Chieffi ${ }^{5}$, M. Limongi ${ }^{6}$, \\ P. François ${ }^{7}$, E. Pancino ${ }^{1,8}$, E. Friel ${ }^{9}$, A. Bragaglia ${ }^{4}$, G. Tautvaišiené ${ }^{10}$, L. Spina ${ }^{11}$, J. Overbeek ${ }^{9}$, T. Cantat-Gaudin ${ }^{12}$, \\ P. Donati ${ }^{4}$, A. Vallenari ${ }^{12}$, R. Sordo ${ }^{12}$, F. M. Jiménez-Esteban ${ }^{13,14}$, B. Tang ${ }^{15}$, A. Drazdauskas ${ }^{10}$, S. Sousa ${ }^{16}$, \\ S. Duffau ${ }^{17,18}$, P. Jofré ${ }^{19,20}$, G. Gilmore ${ }^{19}$, S. Feltzing ${ }^{21}$, E. Alfaro ${ }^{22}$, T. Bensby ${ }^{21}$, E. Flaccomio ${ }^{23}$, S. Koposov ${ }^{19}$, \\ A. Lanzafame ${ }^{24}$, R. Smiljanic ${ }^{25}$, A. Bayo ${ }^{26,27}$, G. Carraro ${ }^{28}$, A. R. Casey ${ }^{19}$, M. T. Costado ${ }^{22}$, F. Damiani ${ }^{23}$, \\ E. Franciosini ${ }^{1}$, A. Hourihane ${ }^{19}$, C. Lardo $^{29}$, J. Lewis ${ }^{19}$, L. Monaco ${ }^{30}$, L. Morbidelli ${ }^{1}$, G. Sacco ${ }^{1}$, L. Sbordone ${ }^{28}$, \\ C. C. Worley ${ }^{19}$, and S. Zaggia ${ }^{12}$ \\ (Affiliations can be found after the references)
}

Received 20 Dedember 2016 / Accepted 1 March 2017

\begin{abstract}
Context. The spatial distribution of elemental abundances in the disc of our Galaxy gives insights both on its assembly process and subsequent evolution, and on the stellar nucleogenesis of the different elements. Gradients can be traced using several types of objects as, for instance, (young and old) stars, open clusters, HII regions, planetary nebulae.

Aims. We aim to trace the radial distributions of abundances of elements produced through different nucleosynthetic channels - the $\alpha$-elements $\mathrm{O}$, $\mathrm{Mg}, \mathrm{Si}, \mathrm{Ca}$ and $\mathrm{Ti}$, and the iron-peak elements $\mathrm{Fe}, \mathrm{Cr}, \mathrm{Ni}$ and $\mathrm{Sc}$ - by use of the Gaia-ESO IDR4 results for open clusters and young-field stars. Methods. From the UVES spectra of member stars, we have determined the average composition of clusters with ages $>0.1$ Gyr. We derived statistical ages and distances of field stars. We traced the abundance gradients using the cluster and field populations and compared them with a chemo-dynamical Galactic evolutionary model.

Results. The adopted chemo-dynamical model, with the new generation of metallicity-dependent stellar yields for massive stars, is able to reproduce the observed spatial distributions of abundance ratios, in particular the abundance ratios of $[\mathrm{O} / \mathrm{Fe}]$ and $[\mathrm{Mg} / \mathrm{Fe}]$ in the inner disc $\left(5 \mathrm{kpc}<R_{\mathrm{GC}}<7 \mathrm{kpc}\right)$, with their differences, that were usually poorly explained by chemical evolution models.

Conclusions. Oxygen and magnesium are often considered to be equivalent in tracing $\alpha$-element abundances and in deducing, for example, the formation timescales of different Galactic stellar populations. In addition, often $[\alpha / \mathrm{Fe}]$ is computed combining several $\alpha$-elements. Our results indicate, as expected, a complex and diverse nucleosynthesis of the various $\alpha$-elements, in particular in the high metallicity regimes, pointing towards a different origin of these elements and highlighting the risk of considering them as a single class with common features.
\end{abstract}

Key words. Galaxy: abundances - open clusters and associations: general

\section{Introduction}

The distribution of elemental abundances in the Galactic disc provides fundamental constraints on models of galaxy formation and evolution. Open clusters are considered among the best tracers of the overall Galactic metallicity distribution and of the thin disc abundance patterns (e.g. Friel 1995, 2013). This is thanks to the wide range of their ages and distances and to the much higher accuracy with which these quantities can be measured in clusters with respect to field stars. The advent of multi-object highresolution spectrometers has allowed us to easily obtain spectra of many stars in the same cluster. This permits us to confidently determine abundances in several member stars, and thus to securely relate the composition (including abundances of a large number of elements belonging to different nucleosynthetic channels) to a specific location and epoch in the history of our Galaxy. Among the several ongoing spectroscopic surveys, the GaiaESO Survey (GES, Gilmore et al. 2012; Randich et al. 2013), a ESO large public survey, is providing high resolution spectra of different stellar population of our Galaxy using VLT/FLAMES

\footnotetext{
* Tables A.1-A.4 are also available at the CDS via anonymous ftp to cdsarc.u-strasbg. fr (130.79.128.5) or via http://cdsarc.u-strasbg.fr/viz-bin/qcat?J/A+A/603/A2
}

(Pasquini et al. 2002), aiming to homogeneously determine stellar parameters and abundances for a large sample of stars in the field and in Galactic open clusters. In particular, in GES the open cluster population is well-sampled and includes clusters over a large range of ages, distances, masses, and metallicities. The cluster target selection will be described in Bragaglia et al. and Randich et al. (in preparation).

Open clusters have historically been used to trace the spatial distribution of metallicity in the Galactic disc. Since the first studies (Janes 1979; Panagia \& Tosi 1980; Janes et al. 1988; Friel \& Janes 1993; Piatti et al. 1995), it has been found that the cluster population shows a significant decrease in metallicity with increasing distance from the Galactic centre, the socalled radial metallicity gradient. Complementary to the study of the overall metallicity distribution (often approximated with $[\mathrm{Fe} / \mathrm{H}])$, the abundance ratios of several elements, such as $\alpha$, neutron-capture, iron-peak and odd-Z elements, can provide insightful information both on the star formation history in the disc and on the nucleosynthesis processes, production sites and timescales of enrichment of each element. For a complete review of the role of open clusters in tracing the Galactic abundance distribution and its time evolution, we refer the reader to Friel (2013). 
In this framework, the behaviour of the so-called $\alpha$-elements - among them $\mathrm{O}, \mathrm{Mg}, \mathrm{Si}, \mathrm{Ca}$, and $\mathrm{Ti}-$ is of particular interest. These elements are indeed mainly formed through stellar nucleosynthetic processes in massive stars. Consequently the timescales at which they are recycled in the interstellar medium is much faster than, for instance, that of iron, which is mainly produced in type Ia supernovae (SNIa). An enhancement of their abundances with respect to iron, or to other iron-peak elements, can reveal differences in the star formation history of different regions of the disc (e.g. Yong et al. 2005). For example, a rapid and intense star formation in the inner disc coupled with a slower and more recent process of star formation in the outer disc, with a still incomplete enrichment by SNIa, might cause an increasing $[\alpha / \mathrm{Fe}]$ in the outer part of the disc. This is indeed predicted by several chemical evolution models (e.g. Magrini et al. 2009, 2015; Kubryk et al. 2013; Minchev et al. 2014). Despite of their common producers in terms of mass range, the creation of the various $\alpha$ elements is related to processes happening during different burning phases in the evolution of massive stars (see, e.g. Pagel \& Tautvaisiene 1995): oxygen is produced during the hydrostatic burning in the He-burning core and in the $\mathrm{C}$-shell and it is expelled during the pre-supernova phase (e.g. Maeder et al. 2005); magnesium is produced during the hydrostatic burning in the $\mathrm{C}$ shell and in the explosive burning of $\mathrm{Ne}$, while the other elements $-\mathrm{Si}, \mathrm{Ca}$ and $\mathrm{Ti}$ - come from the explosive burning.

The existence of such $\alpha$-enhancement in the outer disc remains under debate. The first studies of the abundances of the outermost disc clusters (Yong et al. 2005; Carraro et al. 2004) observed that clusters in the outskirt of the Galaxy had an enhancement in their $\alpha$-element and rapid ( $r$ ) neutron capture elements (e.g. europium). Based on the measured enhancement in $\alpha$ - and $r$-element abundance ratios, Yong et al. (2005) suggested that the outer-disc open clusters were formed in a different way than the rest of the disc, and they proposed their formation through a series of merger events. On the other hand, other works have suggested that abundances of outerdisc open clusters are instead consistent with scaled solar values (e.g. Carraro et al. 2007; Sestito et al. 2008; Pancino et al. 2010; Bensby et al. 2011; Yong et al. 2012; Hayden et al. 2015).

For the same reasons that we expect the $\alpha$-enhancement in the outer parts of the Galactic disc, we foresee that clusters in the very inner disc might present a depletion in $\alpha$ elements over iron with respect to solar values. This is particularly true for the young populations in the inner disc that trace the full chemical evolution of the inner disc characterised by high infall and star-formation rates. However, observations of young populations located in the inner parts of the Galactic disc seem to contradict the expectations of chemical evolution models built in an inside-out scenario (see for example Fig. 9 of Minchev et al. 2014). For example, the observations of "young" $\alpha$-enhanced stars (Chiappini et al. 2015; Martig et al. 2015; Kordopatis et al. 2015; Yong et al. 2016) with $[\mathrm{Fe} / \mathrm{H}]$ ranging from -0.4 to +0.2 dex and located towards the Galactic Centre (see, e.g. Martig et al. 2015; Jofré et al. 2016, for possible explanations of their origin- in the former, young stars formed from gas survived near to the bar with a peculiar composition, in the latter, massive old stars accreted mass from companion) and the hints given by young inner-disc open clusters located at $R_{\mathrm{GC}} \leq 7 \mathrm{kpc}$ (Magrini et al. 2015) are difficult to reconcile with the classical inside-out scenario. For these two young populations, the surprising result is the higher than expected $[\mathrm{Mg} / \mathrm{Fe}]$ value - used as a proxy of $[\alpha / \mathrm{M}]-$ for their age and location in the disc. They are indeed young and presumably born in the inner disc: a sub-solar value of $[\mathrm{Mg} / \mathrm{Fe}]$ should be presumed because of the high infall and high star formation rates in the central part of galaxies (see, e.g. Minchev et al. 2014). Thus even solar $[\mathrm{Mg} / \mathrm{Fe}]$ values alone, as found in the open cluster Be 81 (Magrini et al. 2015), are surprising when compared with what is expected from chemical evolution, that is under-solar $[\mathrm{Mg} / \mathrm{Fe}]$ values (see, e.g. Fig. 9 of Minchev et al. 2014, and Fig. 8 of Kubryk et al. 2015b for oxygen).

In this paper, we make use of the UVES results of both open clusters and Milky Way field stars to investigate the radial trends of several elements, and compare them with the predictions of a chemical evolution model that includes radial migration (Kubryk et al. 2015a,b) and new generation stellar yields for massive stars (see Prantzos et al., in prep., and Limongi $\&$ Chieffi, in prep.). In the following, we indicate the model adopted in the present paper as K15-improved, with the meaning described above. We discuss the differences among the behaviours of the various elements, their implications on the nucleosynthesis in massive stars and SNIa and on the formation of the disc.

The paper is structured as follows: in Sect. 2 we present the data reduction and analysis and in Sect. 3 we determine our solar scale. In Sect. 4 we describe our sample of open clusters and in Sect. 5 the sample of field stars. In Sects. 6 and 7 we show the radial distributions of $[\mathrm{Fe} / \mathrm{H}]$ and of the abundance ratios, and the abundance patterns as function of metallicity. In Sect. 8 we present a chemical evolution whose comparison with the data is presented in Sect. 9. In Sect. 10 we give our summary and conclusions.

\section{Data reduction and analysis}

The UVES spectra used in the present work have been reduced and analysed by the Gaia-ESO consortium in several working groups (WGs). UVES data reduction is carried out using the FLAMES-UVES ESO public pipeline (Modigliani et al. 2004). The UVES data reduction process and the determination of the radial velocities (RVs) are described in Sacco et al. (2014). Different WGs contribute to the spectral analysis of different kinds of stars and/or setups: the data discussed in the present paper have been analysed by WG11. This working group is in charge of the analysis of the UVES spectra of F-G-K spectral type stars both in the field of the Milky Way (MW) and in intermediateage and old clusters, that were obtained with two setups - U580 and U520. The UVES spectra were analysed with the GaiaESO multiple pipelines strategy, as described in Smiljanic et al. (2014). The results of each pipeline are combined with an updated methodology (Casey et al., in prep.) to define a final set of recommended values of the atmospheric parameters. The results of WG11 are homogenised with the results of the other WGs using several calibrators for example, benchmark stars and open/globular clusters selected as described in Pancino et al. (2017) and adopted for the homogenisation by WG15 (Hourihane et al., in prep.). The final recommended stellar parameters of most of the stars included in the fourth internal data release, hereafter IDR4, come from the combination of the results of many Nodes participating to the analysis with different methods, from the equivalent width to the spectral synthesis. In IDR4, ten Nodes were contributing to the analysis of F-G-K UVES spectra. The final recommended parameters of $41 \%$ of the stars are obtained combing the results of all ten nodes, $21 \%$ of nine nodes, $14 \%$ of eight nodes, $9 \%$ of seven nodes, $6 \%$ of six nodes, $4 \%$ of five nodes, $3 \%$ of four nodes, and only $2 \%$ of three or two nodes. The production of the final abundances is a complex process in 
Table 1. IDR4 solar parameters and abundances.

\begin{tabular}{llll}
\hline \hline Sun & $\begin{array}{l}T_{\text {eff }} \\
(\mathrm{K})\end{array}$ & $\log g$ & $\begin{array}{l}\xi \\
\mathrm{km} \mathrm{s}^{-1}\end{array}$ \\
\hline & $5740 \pm 120$ & $4.40 \pm 0.20$ & $0.90 \pm 0.10$ \\
\hline Element & Sun (IDR4*) & Sun (G07) & M 67 giants (IDR4) \\
\hline Fe & $7.48 \pm 0.06$ & $7.45 \pm 0.05$ & $7.48 \pm 0.09(0.02)$ \\
O & $8.78 \pm 0.11$ & $8.66 \pm 0.05$ & $8.76 \pm 0.11(0.02)$ \\
MgI & $7.65 \pm 0.12$ & $7.53 \pm 0.09$ & $7.63 \pm 0.12(0.02)$ \\
SiI & $7.47 \pm 0.07$ & $7.51 \pm 0.04$ & $7.48 \pm 0.07(0.03)$ \\
CaI & $6.31 \pm 0.08$ & $6.31 \pm 0.04$ & $6.31 \pm 0.08(0.02)$ \\
ScII & $3.21 \pm 0.07$ & $3.17 \pm 0.10$ & $3.21 \pm 0.07(0.01)$ \\
TiI & $4.89 \pm 0.08$ & $4.90 \pm 0.06$ & $4.89 \pm 0.08(0.03)$ \\
VI & $3.89 \pm 0.09$ & $4.00 \pm 0.02$ & $4.00 \pm 0.08(0.03)$ \\
CrI & $5.60 \pm 0.10$ & $5.64 \pm 0.10$ & $5.58 \pm 0.11(0.02)$ \\
NiI & $6.23 \pm 0.09$ & $6.23 \pm 0.04$ & $6.24 \pm 0.10(0.01)$ \\
\hline
\end{tabular}

Notes. Average of the several measurements on the different solar U580 spectra from the WG11 analysis.

which all nodes give for each element the abundance line by line. The node abundances (line by line) were combined to produce a final homogenised abundance (per line, per star), which are in turn combined to produce a final recommended abundance per star. Thus it is not straighford to keep trace of the exact lines used to produce the final abundance in each stars. The full linelist (used mainly for spectral synthesis) and the "clean" line-list (used mainly for equivalent width analysis), together with the source and selection of the $\log g f$, which are both experimental or theoretical, preferring, when available, the most precise laboratory measurements, are described in Heiter et al. (2015) and will be available in a forthcoming paper (Heiter et al. in preparation). In the following analysis, we discuss abundances normalised to our internal solar scale, thus mitigating the effect of the $\log g f$ choice in the comparison with literature results.

The recommended parameters and abundances used in the present work are reported in the final GESIDR4FINAL catalogue, which contains the observations obtained until July 2014 and which is distributed to the whole community through the ESO portal.

\section{Solar abundance scale}

To obtain abundances on the solar scale, we need to define our abundance reference. In Table 1 we show the solar parameters (derived in a homogeneous way as the whole IDR4 sample combining the results of the node participating to the analysis) and three different sets of abundances. The solar abundances from IDR4, the Grevesse et al. (2007) ones, and the abundances of giant stars in M67. The cluster M67 is indeed known to have the same composition as the Sun (e.g. Pasquini et al. 2008; Önehag et al. 2014; Liu et al. 2016) and thus it is useful to confirm it with the GES IDR4 data. Furthermore we aim at checking the presence of any systematic difference between the abundances obtained for dwarf and giant stars. We have obtained our reference solar abundances from the average values of all UVES abundance determinations (from the WG11 recommended table) in the same setup used for our science observations, U580. We have also compared them with the reference solar abundances from Grevesse et al. (2007) finding a very good agreement for most elements. Oxygen and magnesium are both slightly higher in the GES Sun. GES oxygen abundance is, however, in good agreement with the results of Caffau et al. (2008) and of Steffen et al. (2015) both based on the [OI] $630.0 \mathrm{~nm}$ line, which is not affected by NLTE and 3D effects. In addition, we report the average abundances of the three member giant stars in M 67 ( $T_{\text {eff }} \sim 4800-4900$ and $\left.\log g \sim 3-3.4\right)$ from the IDR4 recommended table. We quote both the errors on the measurement (from the IDR4 recommended table) and the standard deviation of the average (in parenthesis). The very small standard deviation indicates a high degree of homogeneity of the cluster and high quality of the results.

The results shown in Table 1 indicate an identical composition of the Sun and of M67 giant stars within the uncertainties, and no evident differences between abundances in dwarf and giant stars. Moreover the higher GES abundances of $\mathrm{O}$ and $\mathrm{Mg}$ than the ones of Grevesse et al. (2007) are confirmed also in M67 and are likely related to the choice of the atomic data and line list for these elements. In the sections below, we normalise our abundances to the solar abundances, computed as the average of several determination from the WG11 recommended table. These are shown in the first column of Table 1.

\section{The cluster sample}

We considered the sample of clusters with ages $>0.1 \mathrm{Gyr}$ whose parameters and abundances have been delivered in IDR4. The sample includes several new clusters released for the first time in IDR4: NGC 2243, Berkeley 25, NGC 6005. NGC 6633, NGC 6802, NGC 2516, Pismis 18 and Trumpler23 and four clusters already processed in previous data releases and discussed in previous papers: Berkeley 81, NGC 4815, Trumpler 20, and NGC 6705. Detailed analysis of NGC 6802 and of Trumpler23 from IDR4 data are presented by Tang et al. (2017) and by Overbeek et al. (2017), respectively. The radial metallicity - expressed by $[\mathrm{Fe} / \mathrm{H}]$ - distribution of the inner disc clusters is discussed in Jacobson et al. (2016), while the gradient traced by the very young clusters and star-formation regions is discussed by Spina et al. (2017). Most of our sample clusters are younger than 2 Gyr, and only the two outermost clusters, NGC 2243 and Berkeley 25 are older than 2 Gyr (see Table 2 for parameters and abundances of the clusters). The population of young and intermediate-age open clusters is extremely useful to trace the recent chemical evolution of the Galactic disc since it is not strongly affected by radial migration (see, e.g. Minchev et al. 2014) and it is the dominant component of the young population in the disc.

In Table 2 we summarise the basic properties of the sample clusters - coordinates, ages, Galactocentric distances, heights above the plane, mean radial velocities of cluster members, median metallicity and the number of cluster member stars used to compute the metallicity and the abundances. For clusters in common, we adopt the same ages and distances as in Jacobson et al. (2016). For the two clusters not previously analysed in GES papers, we adopt distance from the Sun from literature studies, and we re-compute Galactocentric distances and heights with $R_{0}=$ $8 \mathrm{kpc}$. For each cluster we have extracted member stars using the information on their radial velocities considering as member stars those within $1 \sigma$ from the cluster systemic velocity and excluding outliers in metallicity $\left|[\mathrm{Fe} / \mathrm{H}]_{\text {star }}-\langle[\mathrm{Fe} / \mathrm{H}]\rangle\right|>0.1$ dex, with a larger range of 0.2 dex allowed for Be81, which has more dispersed member stars in terms of metallicity. For each cluster, based on stars assigned as members, we have computed the median elemental abundances, expressed in the form $12+\log (\mathrm{X} / \mathrm{H})$, which are presented in Table 3 . The error reported on each abundance is the dispersion (computed with the robust sigma) of cluster member abundances. We do not report $12+\log (\mathrm{O} / \mathrm{H})$ in $\operatorname{Tr} 20$ because of telluric contamination of the $[\mathrm{OI}]$ oxygen line 
Table 2. Cluster parameters.

\begin{tabular}{|c|c|c|c|c|c|c|c|c|c|}
\hline \multicolumn{3}{|c|}{$\mathrm{J} 2000.0$} & $\begin{array}{l}\text { Age } \\
\text { (Gyr) }\end{array}$ & $\begin{array}{l}R_{\mathrm{GC}}(\mathrm{a}) \\
(\mathrm{kpc})\end{array}$ & $\begin{array}{l}Z \\
(\mathrm{pc})\end{array}$ & $\begin{array}{l}\mathrm{RV} \\
\left(\mathrm{km} \mathrm{s}^{-1}\right)\end{array}$ & {$[\mathrm{Fe} / \mathrm{H}]$} & n. stars & Ref. age and distance \\
\hline NGC 2516 & $07: 58: 04$ & $-60: 45: 12$ & $0.12 \pm 0.04$ & $7.98 \pm 0.01$ & $-97 \pm 4$ & $+23.6 \pm 1.0$ & $+0.06 \pm 0.05$ & 13 & Sung et al. (2002) \\
\hline NGC 6705 & $18: 51: 05$ & $-06: 16: 12$ & $0.30 \pm 0.05$ & $6.33 \pm 0.16$ & $-95 \pm 10$ & $+34.9 \pm 1.6$ & $+0.12 \pm 0.05$ & 15 & Cantat-Gaudin et al. (2014) \\
\hline NGC 4815 & $12: 57: 59$ & $-64: 57: 36$ & $0.57 \pm 0.07$ & $6.94 \pm 0.04$ & $-95 \pm 6$ & $-29.6 \pm 0.5$ & $+0.00 \pm 0.04$ & 3 & Friel et al. (2014) \\
\hline NGC 6633 & $18: 27: 15$ & $+06: 30: 30$ & $0.63 \pm 0.10$ & $7.71 \pm 0.01$ & $+52 \pm 2$ & $-28.8 \pm 1.5$ & $-0.06 \pm 0.06$ & 8 & Jeffries et al. (2002) \\
\hline NGC 6802 & $19: 30: 35$ & $+20: 15: 42$ & $1.00 \pm 0.10$ & $6.96 \pm 0.07$ & $+36 \pm 3$ & $+11.9 \pm 0.9$ & $+0.10 \pm 0.02$ & 8 & Jacobson et al. (2016) \\
\hline $\operatorname{Be} 81$ & $19: 01: 36$ & $-00: 31: 00$ & $0.86 \pm 0.10$ & $5.49 \pm 0.10$ & $-126 \pm 7$ & $-126 \pm 7$ & $+0.22 \pm 0.07$ & 13 & Magrini et al. (2015) \\
\hline $\operatorname{Tr} 23$ & 16:00:50 & $-53: 31: 23$ & $0.80 \pm 0.10$ & $6.25 \pm 0.15$ & $-18 \pm 2$ & $-61.3 \pm 0.9$ & $+0.14 \pm 0.03$ & 11 & Jacobson et al. (2016) \\
\hline NGC 6005 & $15: 55: 48$ & $-57: 26: 12$ & $1.20 \pm 0.30$ & $5.97 \pm 0.34$ & $-140 \pm 30$ & $-24.1 \pm 1.3$ & $+0.16 \pm 0.02$ & 7 & Piatti et al. (1998) \\
\hline Pis 18 & $13: 36: 55$ & $-62: 05: 36$ & $1.20 \pm 0.40$ & $6.85 \pm 0.17$ & $+12 \pm 2$ & $-27.5 \pm 0.7$ & $+0.10 \pm 0.01$ & 3 & Piatti et al. (1998) \\
\hline $\operatorname{Tr} 20$ & $12: 39: 32$ & $-60: 37: 36$ & $1.50 \pm 0.15$ & $6.86 \pm 0.01$ & $+136 \pm 4$ & $-40.2 \pm 1.3$ & $+0.12 \pm 0.04$ & 27 & Donati et al. (2014) \\
\hline $\mathrm{Be} 44$ & $19: 17: 12$ & $+19: 33: 00$ & $1.60 \pm 0.30$ & $6.91 \pm 0.12$ & $+130 \pm 20$ & $-8.7 \pm 0.7$ & $+0.20 \pm 0.06$ & 4 & Jacobson et al. (2016) \\
\hline $\operatorname{Be} 25$ & $06: 41: 16$ & $-16: 29: 12$ & $4.00 \pm 0.50$ & $17.60 \pm 1.00$ & $-1900 \pm 200$ & $+136.0 \pm 0.8$ & $-0.25 \pm 0.05$ & 6 & Carraro et al. (2005) \\
\hline NGC 2243 & $06: 29: 34$ & $-31: 17: 00$ & $4.00 \pm 1.20$ & $10.40 \pm 0.20$ & $-1200 \pm 100$ & $+60.2 \pm 0.5$ & $-0.38 \pm 0.04$ & 16 & Bragaglia \& Tosi (2006) \\
\hline
\end{tabular}

Table 3. Clusters' elemental abundances expressed in the form $12+\log (\mathrm{X} / \mathrm{H})$.

\begin{tabular}{|c|c|c|c|c|c|c|c|c|c|}
\hline Id & $\mathrm{OI} / \mathrm{H}$ & $\mathrm{MgI} / \mathrm{H}$ & $\mathrm{SiI} / \mathrm{H}$ & $\mathrm{CaI} / \mathrm{H}$ & $\mathrm{TiI} / \mathrm{H}$ & $\mathrm{ScII} / \mathrm{H}$ & $\mathrm{VI} / \mathrm{H}$ & $\mathrm{CrI} / \mathrm{H}$ & $\mathrm{NiI} / \mathrm{H}$ \\
\hline NGC 2516 & - & $7.62 \pm 0.05$ & $7.34 \pm 0.07$ & $6.29 \pm 0.03$ & $4.96 \pm 0.08$ & $3.07 \pm 0.06$ & $3.99 \pm 0.06$ & $5.61 \pm 0.08$ & $6.13 \pm 0.04$ \\
\hline NGC 6705 & $8.75 \pm 0.06$ & $7.85 \pm 0.05$ & $7.59 \pm 0.04$ & $6.37 \pm 0.07$ & $4.93 \pm 0.07$ & $3.20 \pm 0.05$ & $4.05 \pm 0.10$ & $5.65 \pm 0.05$ & $6.34 \pm 0.03$ \\
\hline NGC 4815 & $8.73 \pm 0.05$ & $7.53 \pm 0.06$ & $7.39 \pm 0.09$ & $6.34 \pm 0.11$ & $4.85 \pm 0.03$ & $3.07 \pm 0.06$ & $3.87 \pm 0.03$ & $5.50 \pm 0.01$ & $6.23 \pm 0.11$ \\
\hline NGC 6633 & - & $7.58 \pm 0.03$ & $7.37 \pm 0.05$ & $6.31 \pm 0.05$ & $4.87 \pm 0.06$ & $3.05 \pm 0.04$ & $3.92 \pm 0.08$ & $5.61 \pm 0.06$ & $6.10 \pm 0.05$ \\
\hline NGC 6802 & $8.74 \pm 0.09$ & $7.69 \pm 0.05$ & $7.53 \pm 0.04$ & $6.36 \pm 0.06$ & $4.92 \pm 0.03$ & $3.23 \pm 0.07$ & $3.99 \pm 0.02$ & $5.65 \pm 0.04$ & $6.24 \pm 0.05$ \\
\hline Be 81 & $8.95 \pm 0.13$ & $7.87 \pm 0.06$ & $7.62 \pm 0.06$ & $6.52 \pm 0.05$ & $5.10 \pm 0.08$ & $3.39 \pm 0.05$ & $4.25 \pm 0.09$ & $5.84 \pm 0.07$ & $6.53 \pm 0.09$ \\
\hline $\operatorname{Tr} 23$ & $8.84 \pm 0.07$ & $7.87 \pm 0.07$ & $7.66 \pm 0.05$ & $6.42 \pm 0.07$ & $4.96 \pm 0.07$ & $3.27 \pm 0.06$ & $4.09 \pm 0.06$ & $5.72 \pm 0.07$ & $6.35 \pm 0.06$ \\
\hline NGC 6005 & $8.85 \pm 0.03$ & $7.82 \pm 0.02$ & $7.64 \pm 0.03$ & $6.46 \pm 0.03$ & $5.02 \pm 0.03$ & $3.29 \pm 0.04$ & $4.13 \pm 0.03$ & $5.75 \pm 0.04$ & $6.39 \pm 0.03$ \\
\hline Pis 18 & $8.74 \pm 0.02$ & $7.69 \pm 0.02$ & $7.54 \pm 0.01$ & $6.33 \pm 0.07$ & $4.89 \pm 0.20$ & $3.19 \pm 0.04$ & $4.00 \pm 0.05$ & $5.61 \pm 0.05$ & $6.22 \pm 0.20$ \\
\hline $\operatorname{Tr} 20$ & - & $7.71 \pm 0.04$ & $7.55 \pm 0.06$ & $6.39 \pm 0.03$ & $4.97 \pm 0.03$ & $3.21 \pm 0.06$ & $4.03 \pm 0.05$ & $5.68 \pm 0.04$ & $6.30 \pm 0.05$ \\
\hline $\mathrm{Be} 44$ & $8.84 \pm 0.20$ & $7.91 \pm 0.01$ & $7.73 \pm 0.02$ & $6.49 \pm 0.08$ & $5.13 \pm 0.03$ & $3.34 \pm 0.07$ & $4.24 \pm 0.04$ & $5.97 \pm 0.03$ & $6.45 \pm 0.03$ \\
\hline NGC 2243 & $8.47 \pm 0.08$ & $7.28 \pm 0.04$ & $7.09 \pm 0.06$ & $5.92 \pm 0.04$ & $4.52 \pm 0.06$ & $2.87 \pm 0.05$ & $3.51 \pm 0.08$ & $5.11 \pm 0.07$ & $5.80 \pm 0.05$ \\
\hline Be 25 & $8.90 \pm 0.18$ & $7.44 \pm 0.12$ & $7.26 \pm 0.08$ & $6.04 \pm 0.11$ & $4.69 \pm 0.08$ & $3.05 \pm 0.09$ & $3.70 \pm 0.07$ & $5.28 \pm 0.08$ & $5.96 \pm 0.08$ \\
\hline
\end{tabular}

at $630.0 \mathrm{~nm}$ and $12+\log (\mathrm{O} / \mathrm{H})$ in NGC 2516 and NGC 6633 because of high dispersion with the robust sigma not converging in these two clusters mostly dwarf stars were indeed observed. The stellar parameters, radial velocities and abundances of the selected cluster members are presented in Tables A.1 and A.2.

In Table 4, for each cluster we present the median abundance ratios with their $1 \sigma$ dispersion normalised to the solar scale in Table 1 . We obtained them by computing the median values of the individual $[\mathrm{X} / \mathrm{Fe}]$ in all the selected cluster member stars. These may slightly differ from the simple subtraction of the me$\operatorname{dian}[\mathrm{X} / \mathrm{H}]$ and $[\mathrm{Fe} / \mathrm{H}]$. In Fig. 1 the abundance ratios of the sample clusters are shown in the $[\mathrm{X} / \mathrm{Fe}]$ vs. $[\mathrm{Fe} / \mathrm{H}]$ planes. Individual member stars are shown, together with the $1 \sigma$ dispersion. We note that for most clusters and elements there are small internal dispersions. However for some elements, as for instance oxygen, the dispersion is higher because of intrinsic difficulties in measuring them.

\section{The inner-disc giant and solar neighbourhood dwarf samples}

To complement our cluster sample, we have also considered the full IDR4 database of stellar parameters and abundances, extracting all field stars observed with UVES 580 belonging to the stars in the Milky Way sample, and in particular to the solar neighbourhood sample (GES_TYPE = "GE_MW") and to the inner disc sample (GES_TYPE = "GE_MW_BL"). For these stars, we computed ages and distances. Our method consists of a projection of the stellar parameters on a set of isochrones (Bressan et al. 2012), thus obtaining a simultaneous determination of distance and estimation of age. The details of the method are described in Kordopatis et al. (2011), with the updates of Recio-Blanco et al. (2014) and Kordopatis et al. (2015).
To compare with our cluster sample, we selected stars with age $<5$ Gyr. In addition we selected only stars with $|z|<0.20 \mathrm{kpc}$, thus having a high probability of belonging to the thin disc.

Our final samples contain: 33 stars in the GE_MW sample and 26 stars in the GE_MW_BL sample with ages $<5$ Gyr and belonging to the thin disc population. Their stellar parameters, ages, distance, heights on the Galactic plane and abundances are presented in Tables A.3 and A.4.

These numbers have to be compared with 113 and 109 thin disc stars (defined on the basis of their height, $z$, above the plane, $|z|<0.20 \mathrm{kpc}$ ) of all ages in the GE_MW and GE_MW_BL samples, respectively. Thus only about $30 \%$ of the thin disc stars in the GES IDR4 are younger than 5 Gyr. If we make a more conservative selection, considering only stars younger than 2 Gyr, we have even lower numbers: $13(11 \%)$ and $7(6 \%)$ in the GE_MW and GE_MW_BL samples of thin disc stars, respectively. This highlights how young and intermediate age stars are poorly represented in the field populations and the importance of clusters to characterise the recent abundance distribution in the thin disc. The histograms of the stellar ages of the Milky Way field sample in the thin disc and in the open cluster sample are shown in Fig. 2. In the histogram (Fig. 2) all ages are consistently computed with the projection on isochrones method, so for the open cluster ages can slightly differ from the ages reported in Table 2.

\section{The radial distribution of abundance ratios in clusters and field stars}

Young and intermediate-age open clusters (together with the sample of thin disc field stars with age $<5$ Gyr whose distances and especially ages are, however, much more 
Table 4. Clusters' abundance ratios.

\begin{tabular}{|c|c|c|c|c|c|c|c|c|c|}
\hline Id & {$[\mathrm{OI} / \mathrm{Fe}]$} & {$[\mathrm{MgI} / \mathrm{Fe}]$} & {$[\mathrm{SiI} / \mathrm{Fe}]$} & {$[\mathrm{CaI} / \mathrm{Fe}]$} & {$[\mathrm{TiI} / \mathrm{Fe}]$} & {$[\mathrm{ScII} / \mathrm{Fe}]$} & {$[\mathrm{VI} / \mathrm{Fe}]$} & {$[\mathrm{CrI} / \mathrm{Fe}]$} & {$[\mathrm{Nil} / \mathrm{Fe}]$} \\
\hline NGC 2516 & 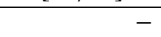 & $0.04 \pm 0.07$ & $-0.04 \pm 0.09$ & $0.01 \pm 0.06$ & $0.13 \pm 0.10$ & $-0.11 \pm 0.08$ & $0.13 \pm 0.08$ & $0.08 \pm 0.09$ & $-0.04 \pm 0.06$ \\
\hline NGC 6705 & $-0.13 \pm 0.07$ & $0.10 \pm 0.07$ & $0.02 \pm 0.07$ & $-0.07 \pm 0.09$ & $-0.04 \pm 0.09$ & $-0.12 \pm 0.07$ & $0.04 \pm 0.11$ & $-0.04 \pm 0.07$ & $0.00 \pm 0.06$ \\
\hline NGC 4815 & $-0.05 \pm 0.05$ & $-0.16 \pm 0.06$ & $-0.05 \pm 0.10$ & $0.05 \pm 0.11$ & $-0.05 \pm 0.04$ & $-0.12 \pm 0.07$ & $0.00 \pm 0.04$ & $-0.10 \pm 0.04$ & $0.02 \pm 0.11$ \\
\hline NGC 6633 & - & $-0.01 \pm 0.07$ & $-0.04 \pm 0.08$ & $0.05 \pm 0.08$ & $0.04 \pm$ & $-0.10 \pm 0.07$ & $0.11 \pm 0.10$ & $0.03 \pm 0.09$ & $-0.08 \pm 0.08$ \\
\hline NGC 6802 & $-0.15 \pm 0.09$ & $-0.05 \pm 0.06$ & $-0.02 \pm 0.05$ & $-0.04 \pm 0.06$ & $-0.07 \pm 0.04$ & $-0.09 \pm 0.08$ & $-0.01 \pm 0.04$ & -0.0 & $-0.0^{7}$ \\
\hline Be81 & $-0.01 \pm 0.13$ & $0.02 \pm 0.09$ & $-0.06 \pm 0.09$ & $0.00 \pm 0.08$ & $0.03 \pm 0.10$ & $-0.05 \pm 0.08$ & $0.15 \pm 0.11$ & 0.10 & \pm 0.11 \\
\hline $\operatorname{Tr} 23$ & -0. & & & -0.0 & -0.0 & -0 . & & -0. & -0.0 \\
\hline NGC 6005 & $-0.09 \pm$ & $0.01 \pm 0.02$ & $-0.01 \pm 0.03$ & $-0.01 \pm 0.03$ & $-0.05 \pm 0.03$ & $-0.09 \pm 0.05$ & $0.09 \pm 0.03$ & \pm 0.04 & -0.02 \\
\hline Pis 18 & $-0.13 \pm 0.03$ & $-0.07 \pm$ & -0.03 & $-0.07=$ & -0.0 & & & -0. & 0.20 \\
\hline $\operatorname{Tr} 20$ & - & $-0.06 \pm$ & -0.04 & -0.0 & -0.0 & -0 . & & -0.0 & -0.0 \\
\hline $\mathrm{Be} 44$ & $-0.05 \pm 0.20$ & $0.06 \pm 0.05$ & $0.06 \pm 0.05$ & $-0.00 \pm 0.09$ & $0.07 \pm 0.06$ & $-0.07 \pm 0.08$ & $0.19 \pm 0.06$ & $0.22 \pm 0.06$ & $0.05 \pm 0.06$ \\
\hline NGC 2243 & & $0.00 \pm 0.06$ & $0.01 \pm 0.07$ & $-0.01 \pm 0.06$ & $0.00 \pm 0.07$ & $0.04 \pm 0.07$ & $-0.01 \pm 0.09$ & $-0.11 \pm 0.08$ & $-0.06 \pm 0.07$ \\
\hline $\operatorname{Be} 25$ & $0.32 \pm 0.18$ & $0.03 \pm 0.13$ & $0.06 \pm 0.09$ & $-0.06 \pm 0.12$ & $0.03 \pm 0.10$ & $0.08 \pm 0.10$ & $0.05 \pm 0.08$ & $-0.05 \pm 0.09$ & $-0.05 \pm 0.09$ \\
\hline
\end{tabular}

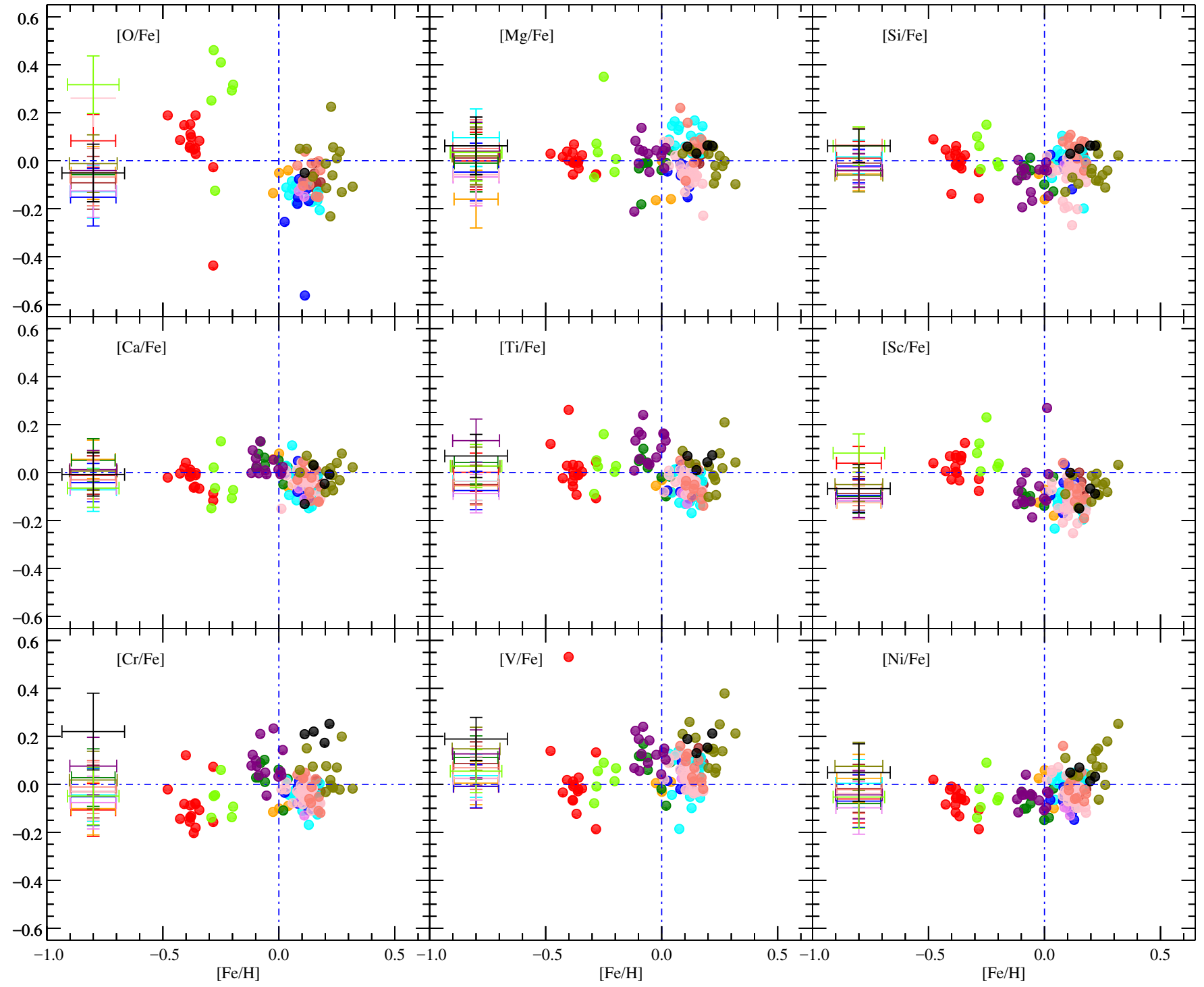

Fig. 1. Abundance ratios of the member stars in our sample clusters. Colour code is the following: NGC 2243 red, NGC 4815 orange, NGC 6005 brown, NGC 6633 green, NGC 6705 cyan, NGC 6802 blue, Pismis 18 violet, Trumpler 20 pink, Trumpler 23 salmon, Be 81 olive, NGC 2516 purple, Be 25 light green, Be 44 black. The crosses on the left side of each panel represents the $1 \sigma$ dispersion of the [Fe/H] and of the abundance ratios.

uncertain) represent a unique (and sometimes neglected) constraint to the shape of the abundance spatial distributions at recent epochs. A number of studies, including the recent results of the Apache Point Observatory Galactic Evolution Experiment (APOGEE) sample (Hayden et al. 2014, 2015), have shown the spatial distributions of the abundances and abundance ratios of field stars (for instance, radial and vertical gradients and azimuthal variations). However, these studies are mainly based on field stars, representative of older populations and consequently they are affected by radial migration. Open clusters 


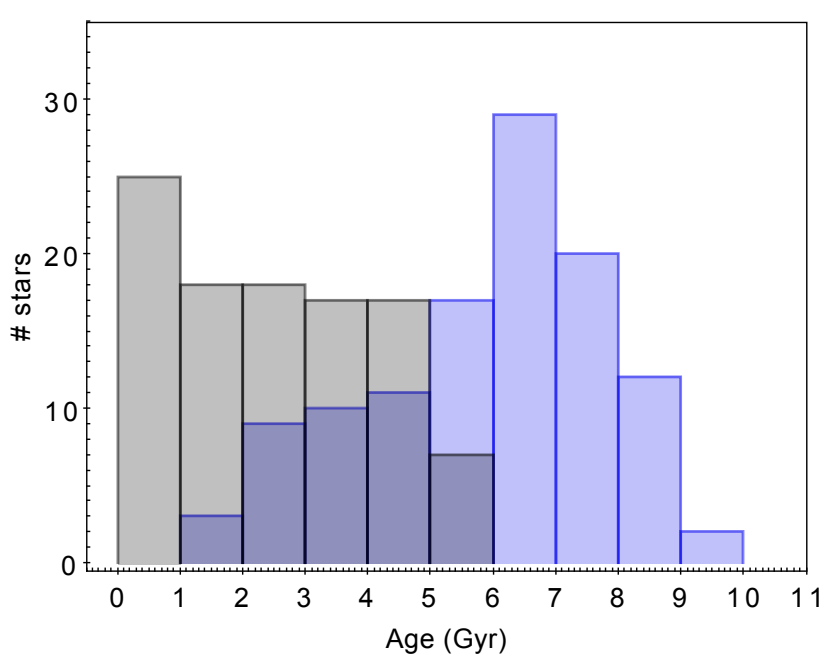

Fig. 2. Stellar ages in the GES Milky Way field sample in the thin disc (in blue) and in the open cluster sample (in grey).

are a valuable alternative tool to study them, being on average younger, and therefore a better tracer of the gradients in the disc out of which the most recent stars formed, as also shown in the recent APOGEE works on open cluster radial metallicity gradient (Frinchaboy et al. 2013; Cunha et al. 2016).

In Fig. 3, we present for our cluster and field star samples the radial abundance ratio distributions of the $\alpha$-elements $[\mathrm{O} / \mathrm{Fe}],[\mathrm{Mg} / \mathrm{Fe}],[\mathrm{Si} / \mathrm{Fe}],[\mathrm{Ca} / \mathrm{Fe}],[\mathrm{Ti} / \mathrm{Fe}]$ and of the iron peakelements $[\mathrm{Sc} / \mathrm{Fe}],[\mathrm{Cr} / \mathrm{Fe}],[\mathrm{V} / \mathrm{Fe}]$ and $[\mathrm{Ni} / \mathrm{Fe}]$. Plotting the abundance ratio over iron allow us to better appreciate the differences between the radial behaviour of each element. The smaller circles in Fig. 3 show the distribution of elemental abundances of thin disc field stars - with ages lower than $5 \mathrm{Gyr}$ - in the IDR4 UVES sample.

The measurement of the oxygen abundance is based on a single absorption line at $\lambda 630.0 \mathrm{~nm}$, and thus it is quite difficult to obtain it, especially in dwarf stars. For this reason, in Fig. 3 we do not plot $[\mathrm{O} / \mathrm{Fe}]$ of dwarf field stars. Even considering only giant stars, the sample of field stars is indeed quite dispersed, whereas the open clusters seem to define an increasing trend towards the outer regions of the disc. The inner-disc clusters have, on average, sub-solar $[\mathrm{O} / \mathrm{Fe}]$, while the two outer-disc clusters reach positive values of $[\mathrm{O} / \mathrm{Fe}]$.

The abundance ratio $[\mathrm{Mg} / \mathrm{Fe}]$ is almost flat all over the disc, with a hint of increasing $[\mathrm{Mg} / \mathrm{Fe}]$ in the inner disc. No suggestions of $\mathrm{Mg}$-enhancement in the outer disc, nor of $\mathrm{Mg}$-depletion in the inner disc are evident from our data. $\mathrm{Si}, \mathrm{Ca}$, and $\mathrm{Ti}$ have all similar behaviours: they reach null values in the inner disc and they are enhanced $(0.05-0.2 \mathrm{dex})$ in the outer disc. For the ironpeak elements, from $\mathrm{Sc}$ to $\mathrm{Ni}$, we find that $[\mathrm{Sc} / \mathrm{Fe}]$ has a slight increases in the outer-disc, similarly to the $\alpha$-elements, while $\mathrm{Cr}$, $\mathrm{V}$ and $\mathrm{Ni}$ are almost constant at solar values across the whole disc.

\section{Abundance patterns of clusters and field stars}

A classic alternative way to look at the Galactic chemical evolution is to consider the behaviour of the abundance ratios versus the iron abundance as a (non linear) proxy of time. In Fig. 4, we show the abundance patterns in the $[\mathrm{X} / \mathrm{Fe}]$ vs. $[\mathrm{Fe} / \mathrm{H}]$ planes of the cluster and thin disc population. For the thin disc, we include stars of all ages to explore also the low metallicity regime. However, the field sample is limited to the solar neighbourhood by the GES selection function (see Stonkutè et al. 2016) and thus it does not reach much lower than $[\mathrm{Fe} / \mathrm{H}]<-1$ dex. The first five panels (from the left) show the abundance patterns of the $\alpha$-elements from oxygen to titanium. The observations show differences in the behaviour of the five elements: oxygen has the strongest trend, reaching negative values of $[\mathrm{O} / \mathrm{Fe}]$ at super-solar metallicities, and having positive values in the low metallicity regime. Magnesium in open clusters is essentially flat, while, at the lowest metallicities, the thin disc field stars reach positive values of $[\mathrm{Mg} / \mathrm{Fe}]$. In addition, contrary to oxygen, the trend of both open clusters and field stars with super-solar metallicities indicates a $[\mathrm{Mg} / \mathrm{Fe}]$ consistent with a slightly positive value. The behaviour of $\mathrm{Si}$ is very similar to that of $\mathrm{Mg}$, with a smaller dispersion in field stars with respect to $\mathrm{Mg}$ due to larger number of available lines for this element in the observed spectral range. $[\mathrm{Si} / \mathrm{Fe}]$ is almost flat and slightly above zero in the super-solar metallicity regime $0.0<[\mathrm{Fe} / \mathrm{H}]<0.5$ dex. However, there are some differences in the most metal poor regime sampled by our stars, where at $[\mathrm{Fe} / \mathrm{H}] \sim-0.5$ dex the few field stars reach higher $[\mathrm{Mg} / \mathrm{Fe}]$ than $[\mathrm{Si} / \mathrm{Fe}]$, and show a different behaviour with respect to the two outermost and most metal poor clusters. The differences might be related to the large errors on the determination of the field star ages (see Table A.1) that may lead us to assign them to an incorrect age bin. Calcium in field stars has a well-defined $[\mathrm{Ca} / \mathrm{Fe}]$ enhancement towards the lowest metallicity, while it is almost flat in open clusters. Finally, Ti is very similar to $\mathrm{Ca}$, having however a larger dispersion in both field and cluster stars abundances.

The last four panels show the abundance ratio of some ironpeak elements. Scandium show differences between field star and open cluster abundances. If we consider field stars, [Sc/Fe] is flat across the metallicity range $[-0.5,0.5]$, with the inner disc open clusters have a depleted $[\mathrm{Sc} / \mathrm{Fe}]$ around -0.1 dex. $\mathrm{V}, \mathrm{Cr}$, and $\mathrm{Ni}$ have similar trends, being almost flat, with a slight enhancement in the super-solar regime.

\section{The chemo-dynamical model}

We compare our observational results with the chemical evolution model of Kubryk et al. (2015a), updated with recent sets of stellar yields for stars of low and high masses (see below). The model is described in details in Kubryk et al. (2015a,b, hereafter $\mathrm{K} 15)$. In the following we recall its main features.

The Galactic disc is gradually built up by infall of primordial gas in the potential well of a typical dark matter halo with mass of $10^{12} M_{\odot}$ whose evolution is obtained from numerical simulations. The infall time-scales are shorter in the inner regions, while they increase outwards reaching 7 Gyr at $7 \mathrm{kpc}$. The star formation rate depends on the local surface density of molecular gas and is calculated with the prescriptions of Blitz \& Rosolowsky (2006).

The model takes into account the radial flows of gas driven by a bar formed $6 \mathrm{Gyr}$ ago which pushes gas inwards and outwards of the corotation. Stars, but also clusters (see, e.g. Gustafsson et al. 2016) move radially due to epicyclic motions (blurring) and variation in their guiding radius (churning), (see e.g. Schönrich \& Binney 2009). The innovative aspect of the model is to account for the fact that radial migration moves around not only passive tracers of chemical evolution (i.e. longlived stars, keeping on their photospheres the chemical composition of the gas at the time and place of their birth), but also active agents of chemical evolution, (i.e. long-lived nucleosynthesis sources such as SNIa producing Fe and low mass stars producing $s$-process elements). The K15 version of the model 


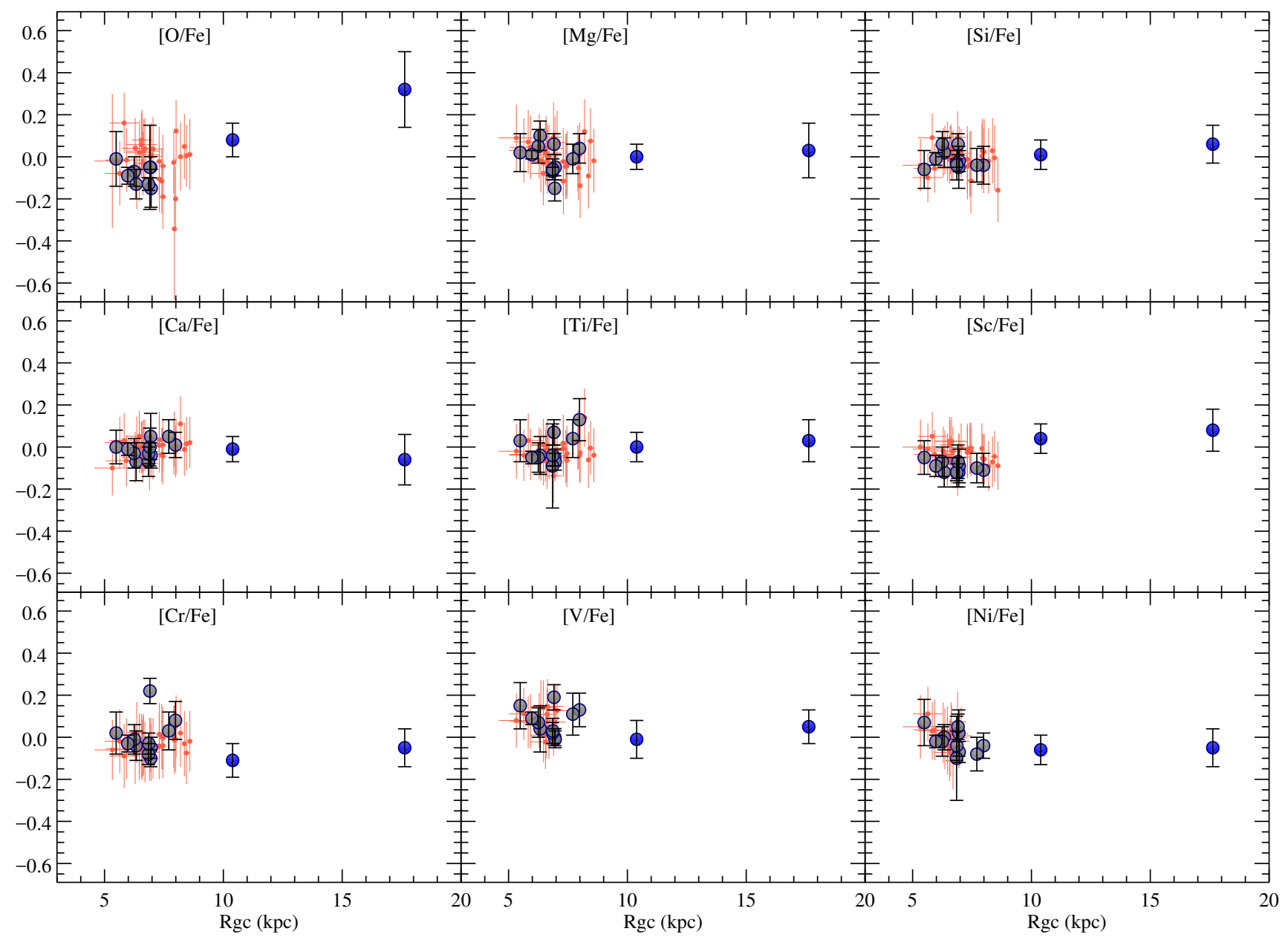

Fig. 3. Radial abundance ratio gradients of elements belonging to different nucleosynthesis channels. The cluster median abundances are shown with larger circles, binned by ages (in grey clusters with age $<2$ Gyr and in blue with age $>2 \mathrm{Gyr}$ ). The smaller red circles are the abundance ratios of the young field stars in the thin disc with their errors.

used for massive stars the metallicity-dependent yields from Nomoto et al. (2013), while the version adopted in the present work (K15-improved) uses the new metallicity-dependent yields by Limongi \& Chieffi (in prep.) which include the effect of stellar rotation.

These yields are based on a new grid of massive stellar models that range in mass between 13 and $120 M_{\odot}$, initial $\mathrm{Fe}$ abundances $[\mathrm{Fe} / \mathrm{H}]=0,-1,-2,-3$ and initial equatorial rotational velocities $v=0,150,300 \mathrm{~km} \mathrm{~s}^{-1}$. The network adopted includes 335 isotopes (from neutrons to $\mathrm{Bi}_{209}$ ) linked by more than 3000 nuclear reactions. The initial composition adopted for the solar metallicity models is the one provided by Asplund et al. (2009), which corresponds to a total metallicity $Z=1.345 \times 10^{-2}$. For metallicities lower than solar we assume a scaled solar distribution for all the elements, with the exception of $\mathrm{C}, \mathrm{O}, \mathrm{Mg}, \mathrm{Si}, \mathrm{S}, \mathrm{Ar}$, $\mathrm{Ca}$, and $\mathrm{Ti}$ which are assumed to be enhanced with respect to $\mathrm{Fe}$. In particular we adopted $[\mathrm{C} / \mathrm{Fe}]=0.18,[\mathrm{O} / \mathrm{Fe}]=0.47$, $[\mathrm{Mg} / \mathrm{Fe}]=0.27,[\mathrm{Si} / \mathrm{Fe}]=0.37,[\mathrm{~S} / \mathrm{Fe}]=0.35, \quad[\mathrm{Ar} / \mathrm{Fe}]=0.35$, $[\mathrm{Ca} / \mathrm{Fe}]=0.33,[\mathrm{Ti} / \mathrm{Fe}]=0.23$ (Cayrel et al. 2004; Spite et al. 2005). As a consequence of these choices, the corresponding metallicity below solar are $Z=3.236 \times 10^{-3}, 3.236 \times 10^{-4}$, $3.236 \times 10^{-5}$, respectively. Stellar rotation has been included as described in detail in Chieffi \& Limongi (2013) with the following exceptions: i) an improved treatment of the angular momentum transport in the envelope of the stars and ii) a detailed computation of the angular momentum loss due to stellar wind. At variance with Chieffi \& Limongi (2013), the efficiency of the rotationally driven mixing has been calibrated by requiring the fit to the observed nitrogen abundance as a function of the projected rotation velocity in the Large Magellanic Cloud samples of the FLAMES survey (Hunter et al. 2009). The explosive nucleosynthesis has been computed in the framework of the kinetic bomb induced explosion by means of a PPM hydrocode, as described in Chieffi \& Limongi (2013). The kinetic energy injected to start the explosion has been calibrated to ejected $0.07 M_{\odot}$ of ${ }^{56} \mathrm{Ni}$ for the models ranging in mass between 13 and $25 M_{\odot}$. This choice leads to final kinetic energies of the ejecta in the range $1-3 \times 10^{51}$ erg that are consistent with the average kinetic energy of a sample of core collapse supernovae reported by Pejcha \& Prieto (2015), Lyman et al. (2016). The explosion of the stars more massive than $25 M_{\odot}$ would require a kinetic energy significantly higher than $3 \times 10^{51}$ erg and we assume that these models fail to explode and collapse directly to a black hole. The yields of these stars therefore contain only the mass ejected through the wind.

A phenomenological rate of SNIa is adopted, based on observations of extragalactic SNIa, while their yields are from Iwamoto et al. (1999). The initial mass function (IMF) of Kroupa (2002), with a slope 1.5 for the high masses, is used. Finally, the formalism of single particle population is used to calculate the rate of ejecta (both for stars and SNIa) as a function 


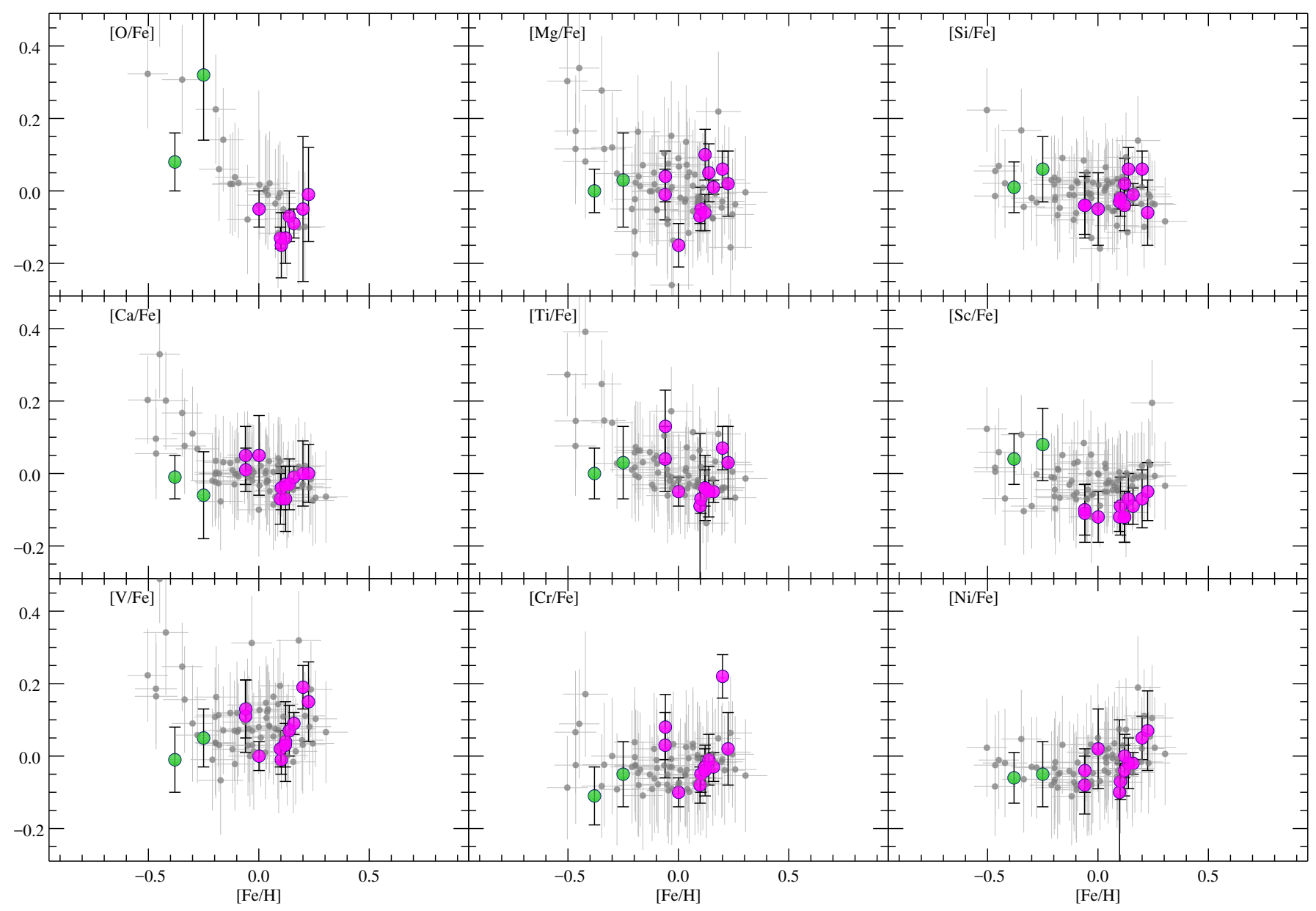

Fig. 4. Abundance patterns of clusters (colour-coded by distance, in magenta with $R_{\mathrm{GC}}<8 \mathrm{kpc}$, and in green with with $R_{\mathrm{GC}}>8 \mathrm{kpc}$ ) and of thin disc field stars (in grey).

of time, because it can account for the radial displacements of nucleosynthesis sources and in particular of SNIa as discussed in Kubryk et al. (2013).

\section{Comparison with the model and discussion}

\subsection{Radial metallicity gradient}

In Fig. 5 we show the radial distribution of $[\mathrm{Fe} / \mathrm{H}]$ of our sample open clusters, colour coded by age: younger than 2 Gyr and older than 2 Gyr. We compare our results with some meaningful samples: the literature compilation of Netopil et al. (2016) (second panel), selecting only open clusters with high resolution observations and determinations of metallicity uncertainties. They are binned in two age bins, similarly to the GES sample: clusters younger than $2 \mathrm{Gyr}$, and clusters with $2 \mathrm{Gyr}<$ Age $<5$ Gyr. In the third panel, we show two other literature open clusters samples: the APOGEE one from Cunha et al. (2016) and the outer-disc clusters' one from Cantat-Gaudin et al. (2016). Finally, in the fourth panel we present the iron abundance of Cepheids (Martin et al. 2015; Genovali et al. 2014, 2015). We compare them with the K15-improved model radial metallicity gradient at there different ages (present-time, 2 Gyr and 5 Gyr ago). Each sample, taken by itself, has its own limit, as for instance, limited statistics (first panel), combination of possible non homogeneous literature results (second panel), possibly poor membership and/or low number of stars per cluster (third panel), and finally large uncertainties on the metallicity determination of each Cepheid (fourth panel). However, there is a general concordance between the shape of the radial metallicity gradients and in the metallicity reached in the four samples. The model curves are a good representation of the general radial behaviour of the $[\mathrm{Fe} / \mathrm{H}]$ in the thin disc of our Galaxy, reproducing the declining gradient at least up to the optical radius of our Galaxy $(\sim 16 \mathrm{kpc})$ from which the open cluster samples show a departure from the negative gradient, reaching a plateau in metallicity (e.g. Sestito et al. 2008; Magrini et al. 2009; Donati et al. 2015; Cantat-Gaudin et al. 2016; Reddy et al. 2016). The plateau is not reproduced by the model and can be related to the high altitude of the outer-disc clusters above the plane, meaning that it is more properly the consequence of a vertical gradient than of a radial gradient and of radial migration and disc flaring (Minchev et al. 2012).

In the first panel, we can see that our sample clusters located within the solar circle $\left(R_{\mathrm{GC}}<8 \mathrm{kpc}\right)$ are all younger than 2 Gyr (the oldest one is Be 44 at $1.6 \mathrm{Gyr}$ ), thus we do not expect that they have moved very far from their birthplace. Anders et al. (2017) considered the possibility that already clusters with ages of about $2 \mathrm{Gyr}$, located from 5 to $8 \mathrm{kpc}$, from the Galactic Centre might be originated from regions located more towards the centre. However, the metallicities of our clusters perfectly follow a radial decreasing gradient (see Fig. 5) with a small dispersion at each Galactocentric radius. If the radial migration were the 


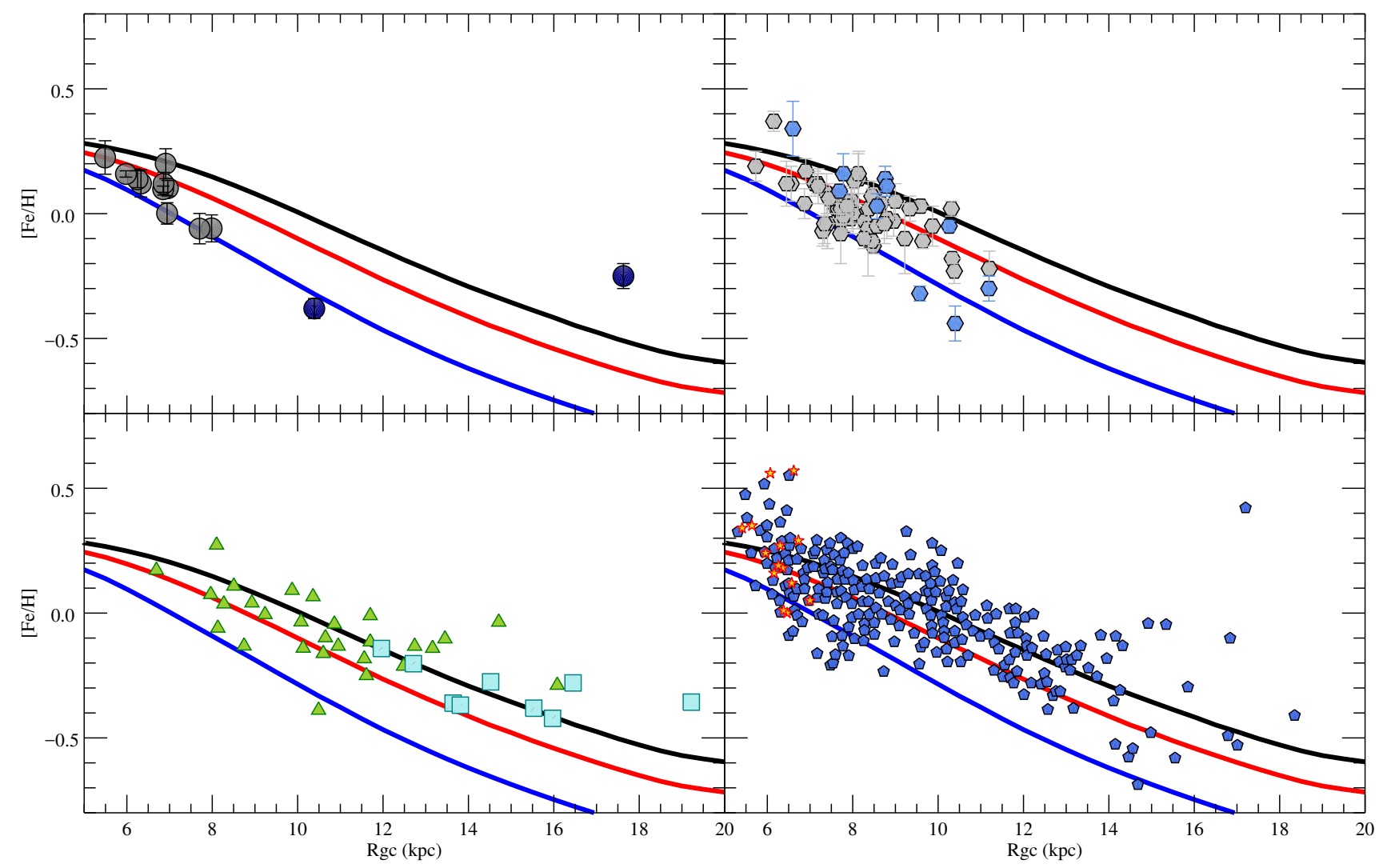

Fig. 5. Radial distributions of $[\mathrm{Fe} / \mathrm{H}]$ for our open cluster sample (first panel: filled circles in grey the youngest clusters, age $<2 \mathrm{Gyr}$, and in blue the oldest ones), for the Netopil et al. (2016)'s open clusters with high-resolution metallicities (second panel: filled hexagons in grey the youngest clusters, age $<2$ Gyr, and in blue the oldest ones), for the APOGEE (Cunha et al. 2016) and Cantat-Gaudin et al. (2016)'s open clusters (third panel: green triangles and cyan squares, resepctively), and for the Cepehids (fourth panel: stars and pentagons Martin et al. 2015; Genovali et al. 2014, 2015, respectively). The black curves represent the gradients of the elements over Fe in the K15-improved model at the present time updated with the new yields of the present work - while the red and blue ones corresponds to 2 Gyr and 5 Gyr ago, respectively.

dominating process, we would expect a very scattered gradient and this is not the case.

Another notable result that can be deduced from Fig. 5 is the unexpected behaviour of the oldest clusters. It is out of the scope of the present paper to discuss the time evolution of the radial metallicity gradient, however as already pointed out in several previous papers (e.g. Jacobson et al. 2016; Anders et al. 2017; Gustafsson et al. 2016; Spina et al. 2017) several old clusters (age $>2 \mathrm{Gyr}$ ) are found to be more metal rich than the younger clusters located at the same Galactocentric radius. There are no old clusters in our sample in the inner disc to compare with the young and intermediate-age ones. However, in the sample of Netopil et al. (2016) there is a super-position of the clusters with ages $>2 \mathrm{Gyr}$ and of the younger ones in the $[\mathrm{Fe} / \mathrm{H}]$ versus $R_{\mathrm{GC}}$ plane, without a clear time-evolution as indicated by the model. In a forthcoming paper (Kawata et al., in prep.), we investigate the effect of radial migration on their location and metallicity.

\subsection{Radial abundance ratio gradient}

In Fig. 6, we present the abundance-ratio radial distributions of the $\alpha$-elements $[\mathrm{O} / \mathrm{Fe}],[\mathrm{Mg} / \mathrm{Fe}],[\mathrm{Si} / \mathrm{Fe}],[\mathrm{Ca} / \mathrm{Fe}],[\mathrm{Ti} / \mathrm{Fe}]$ and of the iron peak-elements $[\mathrm{Sc} / \mathrm{Fe}],[\mathrm{Cr} / \mathrm{Fe}],[\mathrm{V} / \mathrm{Fe}]$ and $[\mathrm{Ni} / \mathrm{Fe}]$ for our clusters. We compare them with the results of the K15improved model curves at the present time and at a look-back time of 5 Gyr.
Oxygen is produced mainly in the nucleosynthesis of massive stars $\left(M>10 M_{\odot}\right)$. These stars have short lifetimes $(<20 \mathrm{Myr})$ that do not give them enough time to migrate. Consequently, the radial $\mathrm{O}$ profile is not affected by radial migration, but, on the other hand, it is strongly impacted by gas radial inflows. The presence of a bar that induces radial gas flows produces a non-monotonic gradient of $[\mathrm{O} / \mathrm{H}]$ as a function of the Galactocentric radius. In the parameterisation of Kubryk et al. (2015a), in the inner regions $(2-4 \mathrm{kpc})$ the combination of the bar and of the metal-poor gas infall leads to a local depression of $[\mathrm{O} / \mathrm{H}]$ with respect to the nearby regions. On the other hand, the disc beyond $6 \mathrm{kpc}$ is not affected by radial inflows, producing a decreasing gradient. In Fig. 6 (first row, first panel to the left) we compare the predictions of the K15-improved model with our observations of clusters and field stars. The data of open clusters seem to support an $[\mathrm{O} / \mathrm{Fe}]$ enhancement in the outer disc for the older clusters, while the conspicuous group of inner disc clusters presents a sub-solar $[\mathrm{O} / \mathrm{Fe}]$ as expected in the model predictions. It is mainly driven by the different timescales for the formation of the inner and outer disc. The ratio between two elements generated by different kinds of stars is able to trace it.

From an observational point of view, the radial distributions of $[\mathrm{Mg} / \mathrm{Fe}],[\mathrm{Si} / \mathrm{Fe}],[\mathrm{Ca} / \mathrm{Fe}]$, and $[\mathrm{Ti} / \mathrm{Fe}]$ shown in Fig. 3 are all very similar, with a slightly increasing trend in the outer disc and solar values in the inner disc. In the model we can distinguish between two kinds of behaviour: $[\mathrm{Mg} / \mathrm{Fe}]$ and $[\mathrm{Ti} / \mathrm{Fe}]$ are essentially flat and do not show any noticeable evolution with time, that is, the radial gradients at the present time and $5 \mathrm{Gyr}$ 


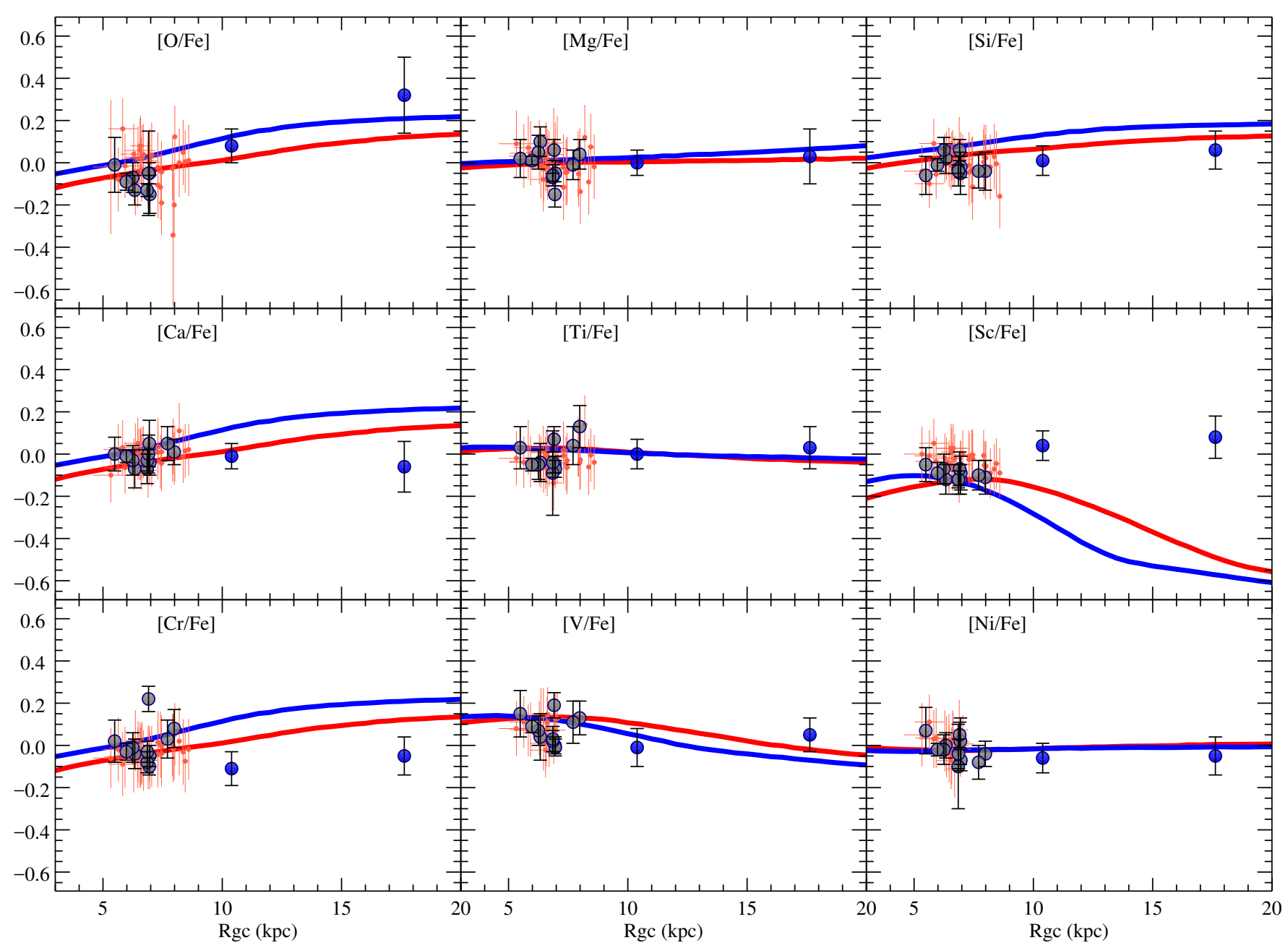

Fig. 6. Radial abundance ratio gradients of elements belonging to different nucleosynthesis channels. For the observations, the symbols are as in Fig. 3. The red curves represent K15-improved model results at the present time, while the blue one corresponds to 5 Gyr ago.

ago are almost similar, while the model results for $[\mathrm{Ca} / \mathrm{Fe}]$ and $[\mathrm{Si} / \mathrm{Fe}]$ show a similar behaviour to $[\mathrm{O} / \mathrm{Fe}]$ with differences between the curves at present time and 5 Gyr ago and an enhancement in the outer disc. The $\alpha$ elements, from Si to the heavier elements $\mathrm{Ca}$ and $\mathrm{Ti}$, are expected to have a non-negligible contribution from SNIa. The yields adopted in the K15 model (the Iwamoto et al. 1999's yields) take the contribution of SNIa to the $\alpha$ elements into account. In addition, the new yields for massive stars used in the current version of the K15 model (Chieffi \& Limongi, in prep.) take into account the stellar rotation and the metallicity dependence.

Comparing with the observations, we have that the model curve of $[\mathrm{Mg} / \mathrm{Fe}]$ is in very good agreement with the observations of both clusters and field stars, and it is very different from the predictions of other chemical evolution models (see, e.g. Minchev et al. 2014). For Si and Ca, while the younger clusters (age $<2$ Gyr) are in good agreement with the model results, the two older and outer-disc clusters do not show the enhancement that is appreciable in $[\mathrm{O} / \mathrm{Fe}]$.

One of the most important results of Fig. 6 is the nice agreement between the observed and modelled radial behaviour of $[\mathrm{Mg} / \mathrm{Fe}]$. This is indeed the first time, to our knowledge, that a chemo-dynamical model is able to distinguish between the evolution of $[\mathrm{O} / \mathrm{Fe}]$ and $[\mathrm{Mg} / \mathrm{Fe}]$. $\mathrm{O}$ and $\mathrm{Mg}$ are considered to have both a predominant production in massive stars. However, they are produced during different burning phases in the evolution of massive stars: oxygen is produced during the hydrostatic burning in the He-burning core and in the C-shell and it is expelled during the pre-supernova phase, in which the final yield can be slightly modified during the explosive Ne burning (see, e.g. Maeder et al. 2005); magnesium is produced during the hydrostatic burning in the $\mathrm{C}$ shell and in the explosive burning of $\mathrm{Ne}$, with a non negligible contribution of this latter process. These differences can explain their abundance distributions.

The radial trend of $\mathrm{Mg}$ is much more similar to that of $\mathrm{Si}, \mathrm{Ca}$ and $\mathrm{Ti}$, and this is presumably an indication of common sites and processes of production. Romano et al. (2010) noticed that the flattening in the $[\mathrm{Si} / \mathrm{Fe}]$ vs. $[\mathrm{Fe} / \mathrm{H}]$ plane traced by observations at super-solar metallicities (Bensby et al. 2005) requires a source of Si enrichment during the latest phases of Galactic chemical evolution. They suggest that this source can be obtained, for instance, from high-metallicity massive stars and/or SNIa.

Introducing the new metallicity dependent yields of massive stars that induce production of elements such as $\mathrm{Mg}$ at recent epochs in the Galaxy lifetime has for the first time reproduced the radial gradient of $[\mathrm{Mg} / \mathrm{Fe}]$ which is essentially flat. A similar conclusion was reached by Romano et al. (2010) who suggested the need for either a revision of current SNII and/or HN yields for solar and/or higher than solar metallicity stars, or larger contributions to $\mathrm{Mg}$ production from SNIa, or significant $\mathrm{Mg}$ synthesis in low- and intermediate-mass stars, or a combination of all these factors. This is in agreement with what was 


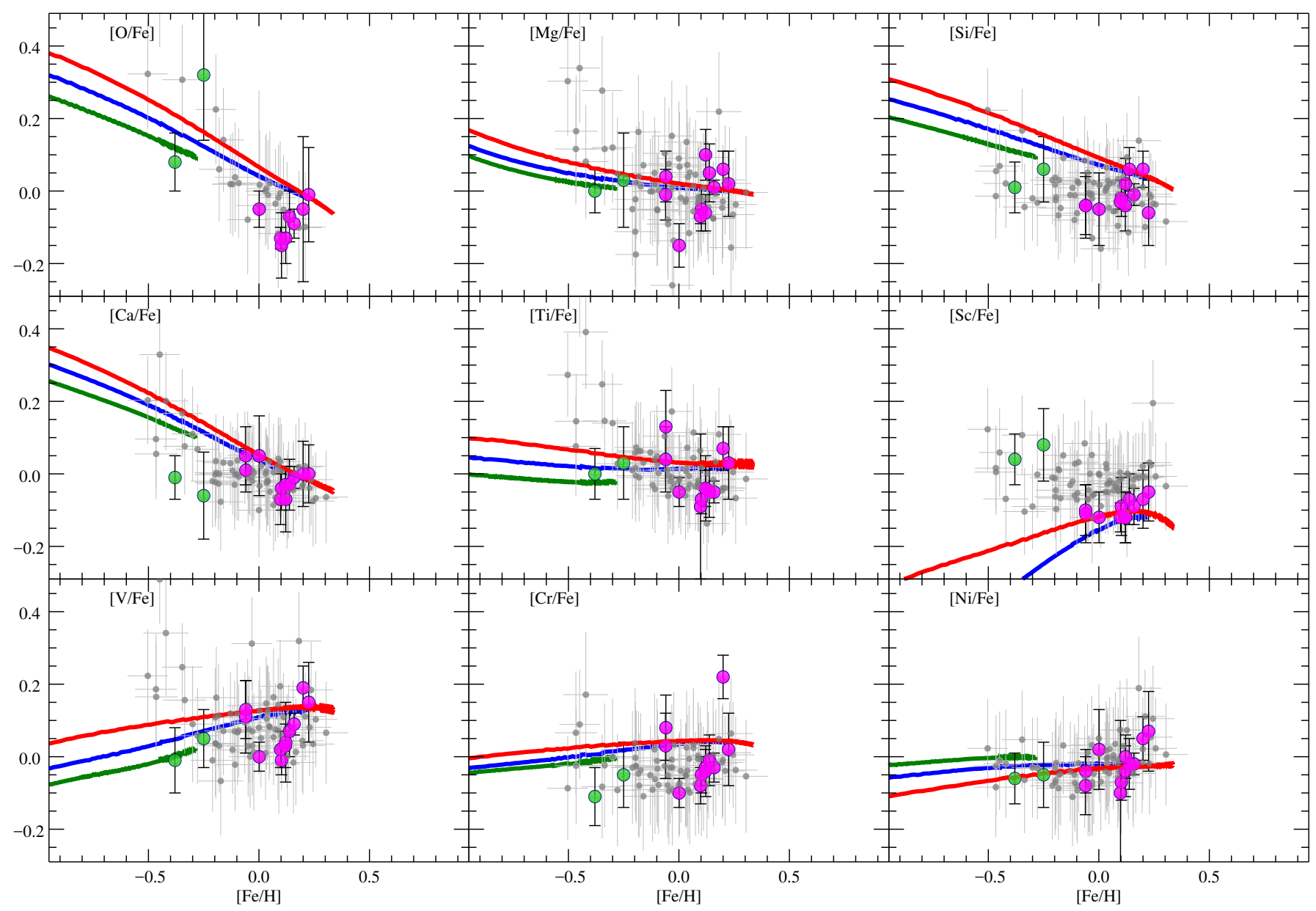

Fig. 7. Abundance patterns of open clusters with the same symbols as in Fig. 4, compared with the curves of the K15-improved model. The model curves are computed for three Galactocentric radii: $6 \mathrm{kpc}$ (red), $8 \mathrm{kpc}$ (blue) and $15 \mathrm{kpc}$ (green).

found by Chiappini (2005) who stated that larger quantities of $\mathrm{Mg}$ (at least a factor of ten larger than current theoretical predictions of either 1D or multi-D models) need to be produced in recent epochs, suggesting a production in SNIa.

The iron-peak group includes many elements ranging from $\mathrm{Sc}$ to $\mathrm{Ge}$ in the periodic table. They are produced in different and complex nucleosynthesis processes that result in a Galactic chemical evolution of their abundances not always following that of iron (cf. Battistini \& Bensby 2015). Here we consider the most representative elements of the iron-group available in our spectral range: $\mathrm{Sc}, \mathrm{V}, \mathrm{Cr}$, and $\mathrm{Ni}$. SNIa contribute very significantly to the iron-peak elements, producing a very little amount of elements lighter than $\mathrm{Al}$ (see, e.g. Iwamoto et al. 1999; Woosley et al. 2009). In addition to the component from $\mathrm{SNIa}, \mathrm{Sc}$ is also produced in the ejected layers of core-collapse $\mathrm{SNe}$ and that its abundance is then further enhanced by neutrinonucleus interactions (Yoshida et al. 2008). Ni, $\mathrm{V}$ and $\mathrm{Cr}$ are also synthesised in massive stars (Woosley \& Weaver 1995; Limongi \& Chieffi 2003). We refer to Romano et al. (2010) for a complete description of the nucleosynthesis processes involved in the production of these elements.

In the final panels of Fig. 6 we see good agreement for $\mathrm{Cr}$, $\mathrm{V}$, and $\mathrm{Ni}$ with the results of K15-improved model. $\mathrm{Cr}$ and, to some extent, $\mathrm{V}$ abundances show a small systematic offset from the predictions, while $\mathrm{Sc}$ is underproduced by the model and the global trend traced by the open clusters is not followed. This indicates that the prescriptions of the model for the nucleosynthesis of Sc need to be updated.

\subsection{Abundance patterns}

In Fig. 7 we present the abundance ratios as a function of the metallicity together with the results of the K15-improved model for three Galactocentric radii: $6 \mathrm{kpc}, 8 \mathrm{kpc}$ and $15 \mathrm{kpc}$. In the case of the $\alpha$-elements, the model predicts two broad behaviours: oxygen, silicon, and calcium have a continuous decreasing trend to super-solar metallicities, reaching negative values for $[\mathrm{O} / \mathrm{Fe}]$ and $[\mathrm{Ca} / \mathrm{Fe}]$, while reaching only zero for $[\mathrm{Si} / \mathrm{Fe}]$. On the other hand, $[\mathrm{Mg} / \mathrm{Fe}]$ and $[\mathrm{Ti} / \mathrm{Fe}]$ become almost flat at $[\mathrm{Fe} / \mathrm{H}] \sim-0.5$. This is caused by two effects: the metallicity dependent yields of massive stars and the contribution of SNIa to their production. For Ti the production in SNIa is the dominant one, while for $\mathrm{Mg}$ the most important contribution is from SNII and the production at later epochs is increased by the metallicity dependent yields of progenitors of SNII. The last four panels show the abundance ratio of the most representative iron-peak elements together with the model predictions. $[\mathrm{Sc} / \mathrm{Fe}]$ is clearly the worst case, in which the model is not able to reproduce the data. $[\mathrm{V} / \mathrm{Fe}],[\mathrm{Cr} / \mathrm{Fe}]$ and $[\mathrm{Ni} / \mathrm{Fe}]$ have similar behaviours, which are, however, not exactly flat. The model is able to reproduce the slight enhancement towards solar and super-solar metallicities that indicate the differences in their nucleosynthesis with that of iron. 
There is a good agreement of the cluster abundance of the inner and outer disc with the corresponding curves of the model: for most elements the outer clusters agree well with outermost plotted curve, while the abundance ratios of the group of nine inner disc clusters are located within the two curves corresponding to 6 and $8 \mathrm{kpc}$. This can explain the differences that can be seen in Fig. 6 in the patterns of field stars and open clusters: while the field star sample is limited to the solar neighbourhood, the open clusters are located in a larger Galactocentric range.

\subsection{On the inside-out scenario for the Galactic thin disc}

The inside-out scenario for the formation of the thin disc predicts a higher star formation rate in the inner parts due to the concurrence of the higher infall rate and of the more effective star formation. The effect of the inside-out formation is appreciable by the presence of negative radial metallicity gradients in most disc galaxies. However, as an effect of different time scales of iron and $\alpha$-elements, we should expect a "positive" gradient of $[\alpha / \mathrm{Fe}]$ in the disc whose entity and slope tell us about the differences in the time scales of the formation of the different regions. The presence of this possible enhancement has been debated for a long time, with contrasting results obtained from different stellar populations: open clusters (see, e.g. Carraro et al. 2004; Yong et al. 2005; Bragaglia et al. 2008), field stars (Carney et al. 2005; Bensby et al. 2011), and Cepheids (Yong et al. 2006). A discussion on this issue using open clusters can be found in Yong et al. (2012).

The problem in many published works has been to use an "average" $\alpha$ - enhancement which is based on different combinations of some of the five more commonly measured elements in the stellar atmospheres of cool stars. The point is, as shown in Figs. 3 and 4, that these five elements do not share the same nucleosynthesis, and this is especially true at solar and super-solar metallicities, which is the characteristics metallicity of the thin disc and it represents the metallicity range spanned by the open cluster population. While it can be acceptable to consider the $\alpha$-elements equivalent to study the dichotomy between the thin and thick discs at low metallicity, they widely diverge from $[\mathrm{Fe} / \mathrm{H}] \sim-0.5$ dex to super solar $[\mathrm{Fe} / \mathrm{H}]$ as appreciable from Figs. 3 and 4. This was already noticed in the seminal work of Pagel \& Tautvaisiene (1995) where those authors already warned about the differences in the nucleosynthesis of the $\alpha$ elements and the risk to mix them in a common $[\alpha / \mathrm{Fe}]$ value.

In Fig. 8 we show the "global" $[\alpha / \mathrm{Fe}]$, computed with $\mathrm{O}, \mathrm{Mg}$, $\mathrm{Si}, \mathrm{Ca}$, and $\mathrm{Ti}$, as function of the $R_{\mathrm{GC}}$ in the model and in the observations. The $[\alpha / \mathrm{Fe}]$ both in model and observations have been computed in the same way, that is, by computing the average of $[\mathrm{X} / \mathrm{Fe}]$ (in some cases some elements are not available, thus the average has been computed with the remaining ones). This is the usual approximation adopted in the various literature studies. The observations are compared with the modelled $[\alpha / \mathrm{Fe}]$ and with $[\mathrm{O} / \mathrm{Fe}]$ : while for $[\mathrm{O} / \mathrm{Fe}]$ we expect in the age interval spanned by open clusters to have in the outer parts of the Galaxy values ranging from $[\mathrm{O} / \mathrm{Fe}]=0.1$ dex to $0.2 \mathrm{dex}$, for $[\alpha / \mathrm{Fe}]$ the expected enhancement is much lower, from 0.05 dex to 0.1 dex. This due to the contribution to the average of elements such as $\mathrm{Ti}$ and $\mathrm{Mg}$ that behave as $\mathrm{Fe}$ at solar and super-solar metallicities. The combination of the five $\alpha$-elements produces an hybrid behaviour that can mask possibly expected differences between inner and outer disc populations.

Thus, our final recommendation is to not use an average $[\alpha / \mathrm{Fe}]$ ratio at least for the typical metallicities of the thin disc. We also suggest distinguishing between the different channels

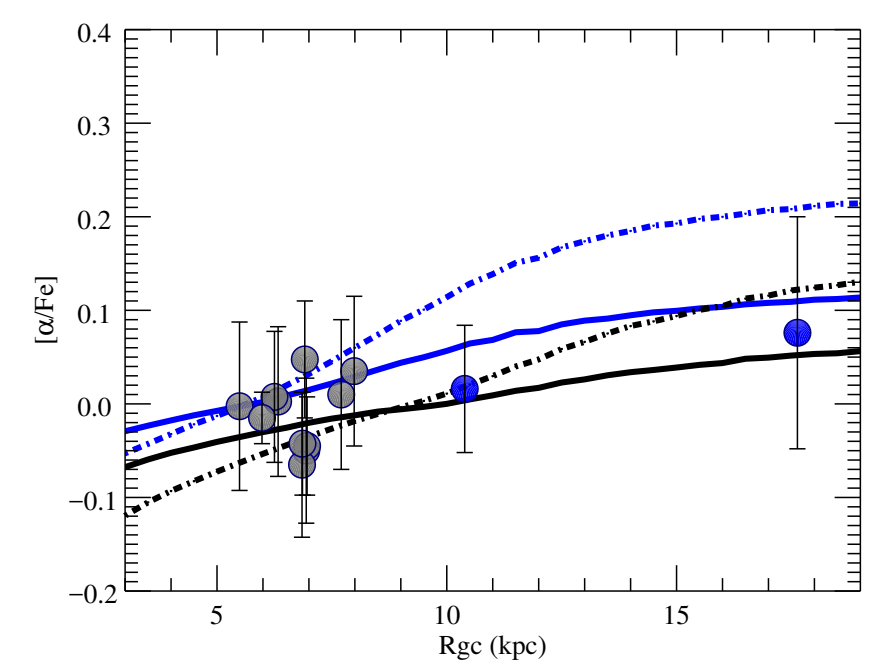

Fig. 8. Global $\alpha$-enhancement: $[\alpha / \mathrm{Fe}]$ vs. $R_{\mathrm{GC}}$ in the open cluster observations (colour coded by age as in Fig. 6) and in the K15-improved model (continuous lines, black at the present time and blue 5 Gyr ago). For comparison, the evolution of $[\mathrm{O} / \mathrm{Fe}]$ vs. $R_{\mathrm{GC}}$ is shown (dasheddotted lines, black at the present time and blue $5 \mathrm{Gyr}$ ago).

of production of the different $\alpha$-elements when seeking subtle trends as the outer disc $\alpha$-enhancement or the inner disc $\alpha$-depletion.

\section{Summary}

We analysed a sample of young and intermediate-age open clusters (age > 0.1 Gyr) in the fourth data release of the Gaia-ESO Survey. Using the recommended stellar parameters and elemental abundances of stars observed with UVES, we determined the median abundances of each cluster. We determine statistical ages and distances of field stars observed with UVES and we selected a sample of stars in the same age range of clusters. Using cluster and field star abundances, we derived the radial distribution of abundance ratios of several $\alpha$ - and iron-peak elements, and their patterns as a function of metallicity, $[\mathrm{Fe} / \mathrm{H}]$. We notice important differences in the diverse classes of elements: in particular we find that $[\mathrm{O} / \mathrm{Fe}]$ has a different behaviour with respect to the other $\alpha$ elements, in particular Mg. We compared our observations, together with literature data, with the results of chemical evolution models that include stellar migration and an updated set of stellar yields for massive stars. The model is able to reproduce the differences in the evolution of $\mathrm{O}$ and $\mathrm{Mg}$, which are usually neglected but that are important especially in the solar and super-solar metallicity regime.

Finally we recommend not using an average $[\alpha / \mathrm{Fe}]$ ratio at least for the typical metallicities of the thin disc. It is necessary to differentiate the channels of production of the $\alpha$-elements when searching for small trends such as the inner disc $\alpha$-depletion and the outer disc $\alpha$-enhancement.

Acknowledgements. The authors thanks the referee for her/his constructive report and Dr. Laura Inno for useful discussions. The results presented here benefited from discussions in three Gaia-ESO workshops supported by the ESF (European Science Foundation) through the GREAT (Gaia Research for European Astronomy Training) Research Network Program (Science meetings 3855, 4127 and 4415). This work was partially supported by the Gaia Research for European Astronomy Training (GREAT-ITN) Marie Curie network, funded through the European Union Seventh Framework Programme [FP7/2007-2013] under grant agreement No. 264895. This work was partly supported through the European Research Council grant 320360: The Gaia-ESO Milky Way Survey. G.T. and A.D. acknowledge support by the Research Council of Lithuania (MIP082/2015). This research has been partially supported by the National Institute for 
Astrophysics (INAF) through the grant PRIN-2014 ("Transient Universe, unveiling new types of stellar explosions with PESSTO"). F.J.E. acknowledges financial support from the Spacetec-CM project (S2013/ICE-2822). S.F. and T.B. are supported by the project grant "The New Milky Way" from the Knut and Alice Wallenberg Foundation. Support for S.D. was provided by the Chile's Ministry of Economy, Development, and Tourism's Millennium Science Initiative through grant IC120009, awarded to The Millennium Institute of Astrophysics, MAS.

\section{References}

Asplund, M., Grevesse, N., Sauval, A. J., \& Scott, P. 2009, ARA\&A, 47, 481 Anders, F., Chiappini, C., Minchev, I., et al. 2017, A\&A, 600, A70 Battistini, C., \& Bensby, T. 2015, A\&A, 577, A9

Bensby, T., Feltzing, S., Lundström, I., \& Ilyin, I. 2005, A\&A, 433, 185

Bensby, T., Alves-Brito, A., Oey, M. S., Yong, D., \& Meléndez, J. 2011, ApJ, 735, L46

Bragaglia, A., \& Tosi, M. 2006, AJ, 131, 1544

Bragaglia, A., Sestito, P., Villanova, S., et al. 2008, A\&A, 480, 79

Bressan, A., Marigo, P., Girardi, L., et al. 2012, MNRAS, 427, 127

Blitz, L., \& Rosolowsky, E. 2006, ApJ, 650, 933

Caffau, E., Ludwig, H.-G., Steffen, M., et al. 2008, A\&A, 488, 1031

Cantat-Gaudin, T., Vallenari, A., Zaggia, S., et al. 2014, A\&A, 569, A17

Cantat-Gaudin, T., Donati, P., Vallenari, A., et al. 2016, A\&A, 588, A120

Carney, B. W., Yong, D., Teixera de Almeida, M. L., \& Seitzer, P. 2005, AJ, 130, 1111

Carraro, G., Bresolin, F., Villanova, S., et al. 2004, AJ, 128, 1676

Carraro, G., Geisler, D., Moitinho, A., Baume, G., \& Vázquez, R. A. 2005, A\&A, 442,917

Carraro, G., Geisler, D., Villanova, S., Frinchaboy, P. M., \& Majewski, S. R. 2007, A\&A, 476, 217

Cayrel, R., Depagne, E., Spite, M., et al. 2004, A\&A, 416, 1117

Chiappini, C. 2005, Interacting Binaries: Accretion, Evolution, and Outcomes, AIP Conf. Proc., 797, 476

Chiappini, C., Anders, F., Rodrigues, T. S., et al. 2015, A\&A, 576, L12

Chieffi, A., \& Limongi, M. 2013, ApJ, 764, 21

Cunha, K., Frinchaboy, P. M., Souto, D., et al. 2016, Astron. Nachr., 337, 922

Donati, P., Cantat Gaudin, T., Bragaglia, A., et al. 2014, A\&A, 561, A94

Donati, P., Bragaglia, A., Carretta, E., et al. 2015, MNRAS, 453, 4185

Friel, E. D. 1995, ARA\&A, 33, 381

Friel, E. D. 2013, in Planets, Stars and Stellar Systems, Vol. 5: Galactic Structure and Stellar Populations (Dordrecht: Springer Science+Business Media), 347

Friel, E. D., \& Janes, K. A. 1993, A\&A, 267, 75

Friel, E. D., Donati, P., Bragaglia, A., et al. 2014, A\&A, 563, A117

Frinchaboy, P. M., Thompson, B., Jackson, K. M., et al. 2013, ApJ, 777, L1

Genovali, K., Lemasle, B., Bono, G., et al. 2014, A\&A, 566, A37

Genovali, K., Lemasle, B., da Silva, R., et al. 2015, A\&A, 580, A17

Gilmore, G., Randich, S., Asplund, M., et al. 2012, The Messenger, 147, 25

Grevesse, N., Asplund, M., \& Sauval, A. J. 2007, Space Sci. Rev., 130, 105

Gustafsson, B., Church, R. P., Davies, M. B., \& Rickman, H. 2016, A\&A, 593, A85

Jacobson, H. R., Friel, E. D., Jilkova, L., et al. 2016, A\&A, 591, A37

Janes, K. A. 1979, ApJS, 39, 135

Janes, K. A., Tilley, C., \& Lynga, G. 1988, AJ, 95, 771

Jeffries, R. D., Totten, E. J., Harmer, S., \& Deliyannis, C. P. 2002, MNRAS, 336, 1109

Jofré, P., Jorissen, A., Van Eck, S., et al. 2016, A\&A, 595, A60

Heiter, U., Lind, K., Asplund, M., et al. 2015, Phys. Scr, 90, 054010

Hunter, I., Brott, I., Langer, N., et al. 2009, A\&A, 496, 841

Kordopatis, G., Recio-Blanco, A., de Laverny, P., et al. 2011, A\&A, 535, A107

Kordopatis, G., Wyse, R. F. G., Gilmore, G., et al. 2015, A\&A, 582, A122

Kroupa, P. 2002, Modes of Star Formation and the Origin of Field Populations, ASP Conf. Proc., 285, 86

Kubryk, M., Prantzos, N., \& Athanassoula, E. 2013, MNRAS, 436, 1479

Kubryk, M., Prantzos, N., \& Athanassoula, E. 2015a, A\&A, 580, A127

Kubryk, M., Prantzos, N., \& Athanassoula, E. 2015b, A\&A, 580, A126

Hayden, M. R., Holtzman, J. A., Bovy, J., et al. 2014, AJ, 147, 116

Hayden, M. R., Bovy, J., Holtzman, J. A., et al. 2015, ApJ, 808, 132

Iwamoto, K., Brachwitz, F., Nomoto, K., et al. 1999, ApJS, 125, 439

Limongi, M., \& Chieffi, A. 2003, ApJ, 592, 404

Liu, F., Asplund, M., Yong, D., et al. 2016, MNRAS, 463, 696

Lyman, J. D., Bersier, D., James, P. A., et al. 2016, MNRAS, 457, 328

Magrini, L., Sestito, P., Randich, S., \& Galli, D. 2009, A\&A, 494, 95

Magrini, L., Randich, S., Donati, P., et al. 2015, A\&A, 580, A85

Martig, M., Rix, H.-W., Aguirre, V. S., et al. 2015, MNRAS, 451, 2230
Martin, R. P., Andrievsky, S. M., Kovtyukh, V. V., et al. 2015, MNRAS, 449, 4071

Maeder, A., Meynet, G., \& Hirschi, R. 2005, Cosmic Abundances as Records of Stellar Evolution and Nucleosynthesis, ASP Conf. Ser., 336, 79

Minchev, I., Famaey, B., Quillen, A. C., et al. 2012, A\&A, 548, A127

Minchev, I., Chiappini, C., \& Martig, M. 2014, A\&A, 572, A92

Modigliani, A., Mulas, G., Porceddu, I., et al. 2004, The Messenger, 118, 8

Netopil, M., Paunzen, E., Heiter, U., \& Soubiran, C. 2016, A\&A, 585, A150

Nomoto, K., Kobayashi, C., \& Tominaga, N. 2013, ARA\&A, 51, 457

Önehag, A., Gustafsson, B., \& Korn, A. 2014, A\&A, 562, A102

Overbeek, J. C., Friel, E. D., Donati, P., et al. 2017, A\&A, 598, A68

Pagel, B. E. J., \& Tautvaisiene, G. 1995, MNRAS, 276, 505

Panagia, N., \& Tosi, M. 1980, A\&A, 81, 375

Pancino, E., Lardo, C., Altavilla, G., et al. 2017, A\&A, 598, A5

Pancino, E., Carrera, R., Rossetti, E., \& Gallart, C. 2010, A\&A, 511, A56

Pasquini, L., Avila, G., Blecha, A., et al. 2002, The Messenger, 110, 1

Pasquini, L., Biazzo, K., Bonifacio, P., Randich, S., \& Bedin, L. R. 2008, A\&A, 489, 677

Pejcha, O., \& Prieto, J. L. 2015, ApJ, 806, 225

Piatti, A. E., Claria, J. J., \& Abadi, M. G. 1995, AJ, 110, 2813

Piatti, A. E., Clariá, J. J., Bica, E., Geisler, D., \& Minniti, D. 1998, AJ, 116, 801

Randich, S., Gilmore, G., \& Gaia-ESO Consortium 2013, The Messenger, 154, 47

Recio-Blanco, A., de Laverny, P., Kordopatis, G., et al. 2014, A\&A, 567, A5

Reddy, A. B. S., Lambert, D. L., \& Giridhar, S. 2016, MNRAS, 463, 4366

Romano, D., Karakas, A. I., Tosi, M., \& Matteucci, F. 2010, A\&A, 522, A32

Sacco, G. G., Morbidelli, L., Franciosini, E., et al. 2014, A\&A, 565, A113

Schönrich, R., \& Binney, J. 2009, MNRAS, 399, 1145

Sestito, P., Bragaglia, A., Randich, S., et al. 2008, A\&A, 488, 943

Smiljanic, R., Korn, A. J., Bergemann, M., et al. 2014, A\&A, 570, A122

Spina, L., Randich, S., Magrini, L., et al. 2017, A\&A, 601, A70

Spite, M., Cayrel, R., Plez, B., et al. 2005, A\&A, 430, 655

Steffen, M., Prakapavičius, D., Caffau, E., et al. 2015, A\&A, 583, A57

Stonkutè, E., Koposov, S. E., Howes, L. M., et al. 2016, MNRAS, 460, 1131

Sung, H., Bessell, M. S., Lee, B.-W., \& Lee, S.-G. 2002, AJ, 123, 290

Tang, B., Geisler, D., Friel, E., et al. 2017, A\&A, 601, A56

Woosley, S. E., \& Weaver, T. A. 1995, ApJS, 101, 181

Woosley, S. E., Kerstein, A. R., Sankaran, V., Aspden, A. J., \& Roumlpke, F. K. 2009, ApJ, 704, 255

Yong, D., Carney, B. W., \& Teixera de Almeida, M. L. 2005, AJ, 130, 597

Yong, D., Carney, B. W., Teixera de Almeida, M. L., \& Pohl, B. L. 2006, AJ, 131, 2256

Yong, D., Carney, B. W., \& Friel, E. D. 2012, AJ, 144, 95

Yong, D., Casagrande, L., Venn, K. A., et al. 2016, MNRAS, 459, 487

1 INAF-Osservatorio Astrofisico di Arcetri, Largo E. Fermi, 5, 50125 Firenze, Italy e-mail: laura@arcetri.astro.it

2 Leibniz-Institut fur Astrophysik Potsdam (AIP), An der Sternwarte 16, 14482 Potsdam, Germany

3 Institut d'Astrophysique de Paris; Université Pierre et Marie Curie, 75014 Paris, France

4 INAF, Osservatorio Astronomico di Bologna, via Ranzani 1, 40127 Bologna, Italy

5 INAF, Istituto di Astrofisica e Planetologia Spaziali, via Fosso del Cavaliere 100, 00133 Roma, Italy

6 INAF, Osservatorio Astronomico di Roma, via Frascati 33, 00078 Roma, Italy

7 Paris-Meudon Observatory, 61 avenue de l'Observatoire, 75014 Paris, France

8 ASI Science Data Center, via del Politecnico snc, 00133 Roma, Italy

9 Department of Astronomy, Indiana University, Bloomington, IN 47405, USA

10 Institute of Theoretical Physics and Astronomy, Vilnius University, Saulètekio al. 3, 10222 Vilnius, Lithuania

11 Universidade de Sao Paulo, IAG Departamento de Astronomia, Rua do Matao 1226, 05509-900 Sao Paulo, Brasil

12 Dipartimento di Fisica e Astronomia, Università di Padova, vicolo Osservatorio 3, 35122 Padova, Italy

13 Centro de Astrobiología (INTA-CSIC), Departamento de Astrofísica, PO Box 78, 28691 Villanueva de la Cañada, Madrid, Spain 
14 Suffolk University, Madrid Campus, C/Valle de la Viña 3, 28003 Madrid, Spain

15 Departamento de Astronomía, Casilla, 160-C, Universidad de Concepción, Concepción, Chile

16 Instituto de Astrofisica e Ciencias do espaço -CAUP, Universidade do Porto, Rua das Estrelas, 4150-762 Porto, Portugal

17 Millennium Institute of Astrophysics, Santiago, Chile

18 Pontificia Universidad Católica de Chile, Av. Vicuña Mackenna 4860, 782-0436 Macul, Santiago, Chile

19 Institute of Astronomy, Madingley Road, University of Cambridge, CB3 0HA, UK

${ }^{20}$ Núcleo de Astronomía, Facultad de Ingeniería, Universidad Diego Portales, Av. Ejército 441, Santiago, Chile

${ }^{21}$ Lund Observatory, Department of Astronomy and Theoretical Physics, Box 43, 22100 Lund, Sweden

22 Instituto de Astrofísica de Andalucía (IAA-CSIC), Glorieta de la Astronomía, 18008 Granada, Spain
23 INAF-Osservatorio Astronomico di Palermo, Piazza del Parlamento 1, 90134 Palermo, Italy

${ }^{24}$ Dipartimento di Fisica e Astronomia, Sezione Astrofisica, Universitá di Catania, via S. Sofia 78, 95123 Catania, Italy

25 Nicolaus Copernicus Astronomical Center, Polish Academy of Sciences, ul. Bartycka 18, 00-716 Warsaw, Poland

26 Facultad de Ciencias, Instituto de Física y Astronomía, Universidad de Valparaíso, Av. Gran Bretana 1111, 5030 Casilla, Valparaíso, Chile

27 Max Planck Institut fur Astronomie, Koenigstuhl 17, 69117 Heidelberg, Germany

28 ESO, Alonso de Cordova 3107, 19001 Santiago de Chile, Chile

29 Astrophysics Research Institute, Liverpool John Moores University, 146 Brownlow Hill, Liverpool L3 5RF, UK

30 Departamento de Ciencias Físicas, Universidad Andres Bello, República 220, Santiago, Chile 


\section{Appendix A: Stellar parameters and abundances of individual stars}

In this appendix we present the stellar parameters, distances, ages, heights, radial velocities, and abundances used in the present work. In Table A.1 we present the stellar parameters and radial velocities of the selected member stars in open clusters, while in Table A.2 their elemental abundances. In Table A.3 we show the stellar parameters, ages, distances, and heights on the Galactic plane of the solar neighbourhood sample of field stars and of the inner disc stars. In Table A.4 we present their elemental abundances in the $12+\log (\mathrm{X} / \mathrm{H})$ form. The complete database for DR4 is available online in the ESO portal.

Table A.1. Stellar parameters and radial velocities of selected member stars in open clusters.

\begin{tabular}{|c|c|c|c|c|c|c|}
\hline Cluster & CNAME & $\begin{array}{l}T_{\text {eff }} \\
(\mathrm{K})\end{array}$ & $\log g$ & $\begin{array}{l}{[\mathrm{Fe} / \mathrm{H}]} \\
(\operatorname{dex})\end{array}$ & $\begin{array}{l}\xi \\
\left(\mathrm{km} \mathrm{s}^{-1}\right)\end{array}$ & $\begin{array}{l}\text { Vrad } \\
\left(\mathrm{km} \mathrm{s}^{-1}\right)\end{array}$ \\
\hline $\mathrm{Br} 25$ & $06411039-1628006$ & $5020 \pm 120$ & $2.51 \pm 0.23$ & $-0.28 \pm 0.10$ & $1.81 \pm 0.27$ & 135.1 \\
\hline $\operatorname{Br} 25$ & $06411363-1629172$ & $4620 \pm 140$ & $2.34 \pm 0.25$ & $-0.28 \pm 0.11$ & $1.49 \pm 0.17$ & 135.3 \\
\hline $\operatorname{Br} 25$ & $06411368-1629164$ & $4710 \pm 120$ & $2.55 \pm 0.25$ & $-0.20 \pm 0.11$ & $1.57 \pm 0.05$ & 135.3 \\
\hline Br 25 & $06411660-1628161$ & $4560 \pm 140$ & $2.33 \pm 0.24$ & $-0.29 \pm 0.11$ & $1.56 \pm 0.02$ & 137.1 \\
\hline Br 25 & $06411680-1630203$ & $4800 \pm 140$ & $2.73 \pm 0.26$ & $-0.20 \pm 0.10$ & $1.50 \pm 0.23$ & 135.7 \\
\hline $\operatorname{Br} 25$ & $06412040-1628236$ & $4600 \pm 140$ & $2.33 \pm 0.27$ & $-0.25 \pm 0.13$ & $1.27 \pm 0.08$ & 136.3 \\
\hline $\mathrm{Br} 44$ & $19170732+1930555$ & $5000 \pm 230$ & $3.13 \pm 0.55$ & $0.11 \pm 0.14$ & $1.54 \pm 0.07$ & -7.7 \\
\hline $\mathrm{Br} 44$ & $19170911+1933256$ & $4940 \pm 180$ & $3.06 \pm 0.44$ & $0.20 \pm 0.12$ & $1.60 \pm 0.12$ & -9.1 \\
\hline $\mathrm{Br} 44$ & $19171388+1933333$ & $4996 \pm 180$ & $2.79 \pm 0.46$ & $0.22 \pm 0.13$ & $1.76 \pm 0.17$ & -9.1 \\
\hline $\operatorname{Br} 44$ & $19172208+1933254$ & $4880 \pm 220$ & $2.76 \pm 0.55$ & $0.15 \pm 0.13$ & $1.44 \pm 0.16$ & -8.7 \\
\hline Br 81 & $19013537-0028186$ & $5170 \pm 140$ & $3.30 \pm 0.29$ & $0.27 \pm 0.13$ & $1.69 \pm 0.14$ & 47.5 \\
\hline $\operatorname{Br} 81$ & $19013631-0027447$ & $4990 \pm 140$ & $2.79 \pm 0.29$ & $0.25 \pm 0.11$ & $1.63 \pm 0.12$ & 47.6 \\
\hline $\operatorname{Br} 81$ & $19013651-0027021$ & $4910 \pm 130$ & $3.14 \pm 0.24$ & $0.22 \pm 0.12$ & $1.62 \pm 0.13$ & 48.9 \\
\hline $\mathrm{Br} 81$ & $19013910-0027114$ & $5010 \pm 120$ & $2.96 \pm 0.23$ & $0.12 \pm 0.10$ & $1.49 \pm 0.19$ & 48.8 \\
\hline $\operatorname{Br} 81$ & $19013997-0028213$ & $4970 \pm 150$ & $3.27 \pm 0.26$ & $0.32 \pm 0.10$ & $1.21 \pm 0.20$ & 48.6 \\
\hline $\operatorname{Br} 81$ & $19014004-0028129$ & $4940 \pm 130$ & $2.77 \pm 0.24$ & $0.26 \pm 0.10$ & $1.39 \pm 0.14$ & 48.7 \\
\hline $\operatorname{Br} 81$ & $19014127-0026444$ & $4870 \pm 130$ & $2.98 \pm 0.25$ & $0.23 \pm 0.10$ & $1.38 \pm 0.05$ & 47.2 \\
\hline $\operatorname{Br} 81$ & $19014194-0028172$ & $4950 \pm 130$ & $2.91 \pm 0.27$ & $0.20 \pm 0.10$ & $1.61 \pm 0.12$ & 48.4 \\
\hline $\operatorname{Br} 81$ & $19014228-0027388$ & $4920 \pm 130$ & $2.83 \pm 0.24$ & $0.22 \pm 0.10$ & $1.49 \pm 0.19$ & 48.6 \\
\hline $\operatorname{Br} 81$ & $19014498-0027496$ & $4740 \pm 120$ & $2.66 \pm 0.23$ & $0.09 \pm 0.10$ & $1.51 \pm 0.12$ & 48.3 \\
\hline $\operatorname{Br} 81$ & $19014525-0023580$ & $4970 \pm 120$ & $2.81 \pm 0.23$ & $0.15 \pm 0.10$ & $1.51 \pm 0.18$ & 49.4 \\
\hline $\operatorname{Br} 81$ & $19014769-0025108$ & $4780 \pm 120$ & $2.59 \pm 0.23$ & $0.20 \pm 0.10$ & $1.58 \pm 0.09$ & 48.0 \\
\hline $\operatorname{Br} 81$ & $19015261-0025318$ & $4950 \pm 130$ & $2.91 \pm 0.25$ & $0.23 \pm 0.12$ & $1.58 \pm 0.10$ & 48.6 \\
\hline NGC 2243 & $06290541-3117025$ & $4930 \pm 110$ & $2.51 \pm 0.21$ & $-0.36 \pm 0.09$ & $1.49 \pm 0.05$ & 60.4 \\
\hline NGC 2243 & $06290934-3110325$ & $4870 \pm 110$ & $2.69 \pm 0.22$ & $-0.38 \pm 0.10$ & $1.27 \pm 0.01$ & 61.3 \\
\hline NGC 2243 & 06291101-3120394 & $4840 \pm 110$ & $2.40 \pm 0.22$ & $-0.38 \pm 0.09$ & $1.46 \pm 0.04$ & 60.7 \\
\hline NGC 2243 & $06292300-3117299$ & $5010 \pm 110$ & $2.55 \pm 0.22$ & $-0.43 \pm 0.10$ & $1.43 \pm 0.05$ & 59.8 \\
\hline NGC 2243 & $06292562-3115261$ & $6010 \pm 140$ & $3.57 \pm 0.25$ & $-0.40 \pm 0.11$ & $1.48 \pm 0.11$ & 58.6 \\
\hline NGC 2243 & $06292841-3117174$ & $4200 \pm 110$ & $1.38 \pm 0.22$ & $-0.48 \pm 0.10$ & $1.63 \pm 0.04$ & 59.2 \\
\hline NGC 2243 & 06292939-3115459 & $4990 \pm 120$ & $2.53 \pm 0.22$ & $-0.38 \pm 0.10$ & $1.36 \pm 0.03$ & 59.7 \\
\hline NGC 2243 & $06293009-3116587$ & $4680 \pm 110$ & $2.13 \pm 0.23$ & $-0.41 \pm 0.10$ & $1.47 \pm 0.01$ & 60.3 \\
\hline NGC 2243 & 06293240-3117294 & $4970 \pm 120$ & $2.50 \pm 0.22$ & $-0.37 \pm 0.10$ & $1.49 \pm 0.04$ & 59.1 \\
\hline NGC 2243 & $06293518-3117239$ & $4930 \pm 110$ & $2.79 \pm 0.21$ & $-0.36 \pm 0.10$ & $1.29 \pm 0.02$ & 61.2 \\
\hline NGC 2243 & $06293525-3115470$ & $5030 \pm 110$ & $3.10 \pm 0.22$ & $-0.34 \pm 0.10$ & $1.33 \pm 0.07$ & 60.2 \\
\hline NGC 2243 & $06294022-3114504$ & $5590 \pm 120$ & $3.62 \pm 0.23$ & $-0.28 \pm 0.10$ & $1.26 \pm 0.16$ & 60.1 \\
\hline NGC 2243 & $06294149-3114360$ & $4740 \pm 110$ & $2.43 \pm 0.22$ & $-0.38 \pm 0.10$ & $1.34 \pm 0.04$ & 60.1 \\
\hline NGC 2243 & $06294582-3115381$ & $4950 \pm 120$ & $2.48 \pm 0.22$ & $-0.38 \pm 0.10$ & $1.38 \pm 0.03$ & 60.8 \\
\hline NGC 2243 & $06294621-3116016$ & $5210 \pm 110$ & $2.89 \pm 0.22$ & $-0.28 \pm 0.11$ & $1.31 \pm 0.04$ & 60.3 \\
\hline NGC 2243 & 06295099-3114428 & $4950 \pm 120$ & $2.91 \pm 0.24$ & $-0.36 \pm 0.09$ & $1.38 \pm 0.10$ & 59.4 \\
\hline NGC 2516 & $07541167-6048001$ & $5520 \pm 130$ & $4.32 \pm 0.24$ & $-0.11 \pm 0.11$ & $1.92 \pm 0.23$ & 23.8 \\
\hline NGC 2516 & $07541553-6058079$ & $5510 \pm 130$ & $4.54 \pm 0.27$ & $0.01 \pm 0.12$ & $1.43 \pm 0.01$ & 24.4 \\
\hline NGC 2516 & $07544342-6024437$ & $5550 \pm 110$ & $4.57 \pm 0.23$ & $0.01 \pm 0.10$ & $1.24 \pm 0.14$ & 25.1 \\
\hline NGC 2516 & $07550592-6104294$ & $5810 \pm 130$ & $4.55 \pm 0.24$ & $-0.10 \pm 0.10$ & $1.39 \pm 0.20$ & 23.1 \\
\hline NGC 2516 & $07553236-6023094$ & $5800 \pm 200$ & $4.48 \pm 0.45$ & $0.02 \pm 0.12$ & $1.35 \pm 0.15$ & 24.8 \\
\hline NGC 2516 & $07564410-6034523$ & $5610 \pm 120$ & $4.40 \pm 0.24$ & $-0.06 \pm 0.10$ & $1.31 \pm 0.14$ & 24.3 \\
\hline NGC 2516 & $07573608-6048128$ & $5600 \pm 110$ & $4.52 \pm 0.24$ & $-0.02 \pm 0.09$ & $1.43 \pm 0.19$ & 23.2 \\
\hline NGC 2516 & $07574792-6056131$ & $5560 \pm 120$ & $4.53 \pm 0.22$ & $0.00 \pm 0.09$ & $1.21 \pm 0.09$ & 25.4 \\
\hline NGC 2516 & $07575215-6100318$ & $5300 \pm 120$ & $4.49 \pm 0.24$ & $-0.09 \pm 0.10$ & $1.40 \pm 0.12$ & 24.4 \\
\hline NGC 2516 & $07583485-6103121$ & $6040 \pm 130$ & $4.68 \pm 0.22$ & $-0.08 \pm 0.10$ & $1.41 \pm 0.13$ & 23.5 \\
\hline NGC 2516 & $07584257-6040199$ & $5590 \pm 120$ & $4.43 \pm 0.25$ & $-0.10 \pm 0.11$ & $1.49 \pm 0.18$ & 25.3 \\
\hline NGC 2516 & $08000944-6033355$ & $5900 \pm 120$ & $4.52 \pm 0.23$ & $-0.05 \pm 0.09$ & $1.39 \pm 0.27$ & 23.8 \\
\hline NGC 2516 & $08013658-6059021$ & $5620 \pm 120$ & $4.39 \pm 0.23$ & $-0.12 \pm 0.10$ & $1.49 \pm 0.18$ & 24.2 \\
\hline NGC 4815 & $12575511-6458483$ & $4920 \pm 120$ & $2.48 \pm 0.23$ & $0.00 \pm 0.09$ & $1.61 \pm 0.21$ & -29.3 \\
\hline NGC 4815 & $12575529-6456536$ & $5030 \pm 110$ & $2.63 \pm 0.23$ & $-0.02 \pm 0.09$ & $1.03 \pm 0.07$ & -29.5 \\
\hline NGC 4815 & $12580262-6456492$ & $4930 \pm 110$ & $2.50 \pm 0.22$ & $0.04 \pm 0.10$ & $1.84 \pm 0.13$ & -29.2 \\
\hline NGC 6005 & $15552962-5724408$ & $4970 \pm 120$ & $3.05 \pm 0.22$ & $0.16 \pm 0.09$ & $1.40 \pm 0.05$ & -24.9 \\
\hline NGC 6005 & $15553687-5729569$ & $4920 \pm 120$ & $2.99 \pm 0.21$ & $0.15 \pm 0.09$ & $1.34 \pm 0.04$ & -23.4 \\
\hline NGC 6005 & $15554015-5726002$ & $4870 \pm 120$ & $2.67 \pm 0.23$ & $0.17 \pm 0.10$ & $1.55 \pm 0.07$ & -24.8 \\
\hline NGC 6005 & $15554127-5726574$ & $4910 \pm 120$ & $2.80 \pm 0.22$ & $0.15 \pm 0.10$ & $1.41 \pm 0.08$ & -26.3 \\
\hline NGC 6005 & $15554167-5725533$ & $4910 \pm 120$ & $2.79 \pm 0.23$ & $0.16 \pm 0.10$ & $1.41 \pm 0.04$ & -23.7 \\
\hline NGC 6005 & $15555612-5726539$ & $4940 \pm 110$ & $2.99 \pm 0.23$ & $0.15 \pm 0.09$ & $1.40 \pm 0.06$ & -23.7 \\
\hline NGC 6005 & $15560208-5727418$ & $4940 \pm 120$ & $2.92 \pm 0.23$ & $0.18 \pm 0.09$ & $1.40 \pm 0.03$ & -24.7 \\
\hline
\end{tabular}


Table A.1. continued.

\begin{tabular}{|c|c|c|c|c|c|c|}
\hline Cluster & CNAME & $\begin{array}{l}T_{\text {eff }} \\
(\mathrm{K})\end{array}$ & $\log g$ & $\begin{array}{l}{[\mathrm{Fe} / \mathrm{H}]} \\
(\mathrm{dex})\end{array}$ & $\begin{array}{l}\xi \\
\left(\mathrm{km} \mathrm{s}^{-1}\right)\end{array}$ & $\begin{array}{l}\text { Vrad } \\
\left(\mathrm{km} \mathrm{s}^{-1}\right)\end{array}$ \\
\hline NGC 6633 & $18263404+0637467$ & $5720 \pm 115$ & $4.37 \pm 0.22$ & $-0.09 \pm 0.09$ & $1.13 \pm 0.12$ & -28.9 \\
\hline NGC 6633 & $18263966+0629501$ & $5580 \pm 110$ & $4.36 \pm 0.23$ & $-0.08 \pm 0.09$ & $1.17 \pm 0.06$ & -27.8 \\
\hline NGC 6633 & $18270752+0627556$ & $5740 \pm 110$ & $4.42 \pm 0.23$ & $-0.06 \pm 0.09$ & $1.14 \pm 0.09$ & -27.6 \\
\hline NGC 6633 & $18274267+0639082$ & $6150 \pm 120$ & $3.84 \pm 0.24$ & $0.04 \pm 0.10$ & $1.61 \pm 0.11$ & -27.1 \\
\hline NGC 6633 & $18274725+0640462$ & $5230 \pm 110$ & $4.44 \pm 0.23$ & $-0.06 \pm 0.10$ & $1.09 \pm 0.08$ & -28.3 \\
\hline NGC 6633 & $18280018+0654514$ & $5050 \pm 110$ & $2.80 \pm 0.23$ & $0.02 \pm 0.09$ & $1.40 \pm 0.05$ & -28.7 \\
\hline NGC 6633 & $18280866+0638090$ & $5120 \pm 120$ & $4.52 \pm 0.23$ & $-0.10 \pm 0.11$ & $1.09 \pm 0.05$ & -27.0 \\
\hline NGC 6633 & $18283246+0644404$ & $5900 \pm 120$ & $4.34 \pm 0.22$ & $0.00 \pm 0.09$ & $1.27 \pm 0.09$ & -27.3 \\
\hline NGC 6705 & $18502831-0615122$ & $4560 \pm 120$ & $1.83 \pm 0.23$ & $0.17 \pm 0.11$ & $1.72 \pm 0.16$ & 34.5 \\
\hline NGC 6705 & $18504563-0612038$ & $4670 \pm 130$ & $2.09 \pm 0.23$ & $0.06 \pm 0.10$ & $1.66 \pm 0.26$ & 35.0 \\
\hline NGC 6705 & $18505494-0616182$ & $4670 \pm 120$ & $2.22 \pm 0.23$ & $0.07 \pm 0.10$ & $1.48 \pm 0.15$ & 35.8 \\
\hline NGC 6705 & $18505581-0618148$ & $4470 \pm 130$ & $1.89 \pm 0.23$ & $0.16 \pm 0.11$ & $1.59 \pm 0.15$ & 36.1 \\
\hline NGC 6705 & $18510289-0615301$ & $4730 \pm 120$ & $2.27 \pm 0.22$ & $0.03 \pm 0.09$ & $1.49 \pm 0.17$ & 34.0 \\
\hline NGC 6705 & $18510399-0620414$ & $4630 \pm 110$ & $2.08 \pm 0.23$ & $0.10 \pm 0.10$ & $1.66 \pm 0.15$ & 34.0 \\
\hline NGC 6705 & $18510662-0612442$ & $4750 \pm 120$ & $2.17 \pm 0.23$ & $0.13 \pm 0.10$ & $1.69 \pm 0.22$ & 33.2 \\
\hline NGC 6705 & $18510786-0617119$ & $4750 \pm 130$ & $2.29 \pm 0.25$ & $0.14 \pm 0.11$ & $1.74 \pm 0.20$ & 34.7 \\
\hline NGC 6705 & $18510833-0616532$ & $4830 \pm 130$ & $2.17 \pm 0.31$ & $0.17 \pm 0.10$ & $1.77 \pm 0.08$ & 34.2 \\
\hline NGC 6705 & $18511116-0614340$ & $4310 \pm 130$ & $1.64 \pm 0.23$ & $0.04 \pm 0.10$ & $1.72 \pm 0.19$ & 33.4 \\
\hline NGC 6705 & $18511534-0618359$ & $4780 \pm 130$ & $2.25 \pm 0.23$ & $0.18 \pm 0.11$ & $1.77 \pm 0.25$ & 34.6 \\
\hline NGC 6705 & $18511571-0618146$ & $4640 \pm 120$ & $2.10 \pm 0.23$ & $0.13 \pm 0.10$ & $1.80 \pm 0.19$ & 36.0 \\
\hline NGC 6705 & $18512662-0614537$ & $4330 \pm 120$ & $1.80 \pm 0.23$ & $0.08 \pm 0.10$ & $1.57 \pm 0.14$ & 34.8 \\
\hline NGC 6705 & $18514034-0617128$ & $4670 \pm 120$ & $1.98 \pm 0.23$ & $0.06 \pm 0.09$ & $1.65 \pm 0.22$ & 33.3 \\
\hline NGC 6705 & $18514130-0620125$ & $4640 \pm 120$ & $2.10 \pm 0.22$ & $0.12 \pm 0.10$ & $1.62 \pm 0.09$ & 34.3 \\
\hline NGC 6802 & $19303058+2013163$ & $5090 \pm 110$ & $2.81 \pm 0.22$ & $0.11 \pm 0.10$ & $1.55 \pm 0.22$ & 11.6 \\
\hline NGC 6802 & $19303085+2016274$ & $5030 \pm 160$ & $2.80 \pm 0.33$ & $0.08 \pm 0.12$ & $1.58 \pm 0.05$ & 12.3 \\
\hline NGC 6802 & $19303184+2014459$ & $5050 \pm 120$ & $2.92 \pm 0.23$ & $0.13 \pm 0.10$ & $1.61 \pm 0.19$ & 12.0 \\
\hline NGC 6802 & $19303274+2014498$ & $4940 \pm 120$ & $2.62 \pm 0.23$ & $0.02 \pm 0.10$ & $1.59 \pm 0.16$ & 12.2 \\
\hline NGC 6802 & $19303309+2015442$ & $4800 \pm 120$ & $2.43 \pm 0.22$ & $0.08 \pm 0.10$ & $1.66 \pm 0.08$ & 11.4 \\
\hline NGC 6802 & $19303611+2016329$ & $4960 \pm 120$ & $2.61 \pm 0.23$ & $0.10 \pm 0.10$ & $1.59 \pm 0.08$ & 12.5 \\
\hline NGC 6802 & $19303884+2014005$ & $5060 \pm 120$ & $2.84 \pm 0.25$ & $0.12 \pm 0.10$ & $1.54 \pm 0.11$ & 13.5 \\
\hline NGC 6802 & $19304170+2015224$ & $5180 \pm 120$ & $3.13 \pm 0.23$ & $0.09 \pm 0.10$ & $1.48 \pm 0.04$ & 11.5 \\
\hline Pis 18 & $13365597-6205130$ & $4920 \pm 120$ & $2.55 \pm 0.23$ & $0.09 \pm 0.09$ & $1.69 \pm 0.09$ & -28.1 \\
\hline Pis 18 & $13370523-6206433$ & $4950 \pm 120$ & $2.66 \pm 0.22$ & $0.10 \pm 0.10$ & $1.51 \pm 0.08$ & -27.7 \\
\hline Pis 18 & $13371182-6206030$ & $4900 \pm 120$ & $2.51 \pm 0.22$ & $0.11 \pm 0.10$ & $1.72 \pm 0.07$ & -28.3 \\
\hline $\operatorname{Tr} 20$ & $12383597-6045242$ & $5000 \pm 120$ & $2.97 \pm 0.23$ & $0.14 \pm 0.09$ & $1.51 \pm 0.08$ & -40.2 \\
\hline $\operatorname{Tr} 20$ & $12385807-6030286$ & $4550 \pm 130$ & $2.18 \pm 0.29$ & $0.09 \pm 0.11$ & $1.45 \pm 0.06$ & -40.8 \\
\hline $\operatorname{Tr} 20$ & $12390478-6041475$ & $5000 \pm 120$ & $2.95 \pm 0.22$ & $0.18 \pm 0.09$ & $1.47 \pm 0.07$ & -40.1 \\
\hline $\operatorname{Tr} 20$ & $12390709-6038056$ & $5030 \pm 120$ & $3.14 \pm 0.23$ & $0.10 \pm 0.10$ & $1.27 \pm 0.04$ & -39.8 \\
\hline $\operatorname{Tr} 20$ & $12391002-6038402$ & $4470 \pm 120$ & $2.14 \pm 0.22$ & $0.01 \pm 0.10$ & $1.56 \pm 0.17$ & -40.2 \\
\hline $\operatorname{Tr} 20$ & $12391113-6036528$ & $4970 \pm 110$ & $2.93 \pm 0.22$ & $0.18 \pm 0.10$ & $1.39 \pm 0.11$ & -41.1 \\
\hline $\operatorname{Tr} 20$ & $12391200-6036322$ & $4940 \pm 110$ & $2.83 \pm 0.22$ & $0.15 \pm 0.10$ & $1.33 \pm 0.05$ & -40.9 \\
\hline $\operatorname{Tr} 20$ & $12392636-6040217$ & $4950 \pm 120$ & $2.86 \pm 0.22$ & $0.08 \pm 0.10$ & $1.46 \pm 0.23$ & -40.9 \\
\hline $\operatorname{Tr} 20$ & $12392698-6036053$ & $4830 \pm 120$ & $2.76 \pm 0.24$ & $0.11 \pm 0.10$ & $1.39 \pm 0.10$ & -39.3 \\
\hline $\operatorname{Tr} 20$ & $12393740-6032568$ & $5030 \pm 120$ & $3.00 \pm 0.22$ & $0.14 \pm 0.10$ & $1.40 \pm 0.10$ & -40.6 \\
\hline $\operatorname{Tr} 20$ & $12393781-6039051$ & $4930 \pm 130$ & $2.79 \pm 0.22$ & $0.12 \pm 0.11$ & $1.44 \pm 0.14$ & -39.1 \\
\hline $\operatorname{Tr} 20$ & $12394307-6039193$ & $4880 \pm 120$ & $2.76 \pm 0.23$ & $0.12 \pm 0.09$ & $1.34 \pm 0.05$ & -40.2 \\
\hline $\operatorname{Tr} 20$ & $12394385-6033165$ & $5000 \pm 110$ & $2.87 \pm 0.23$ & $0.09 \pm 0.10$ & $1.58 \pm 0.06$ & -40.1 \\
\hline $\operatorname{Tr} 20$ & $12394418-6034410$ & $4960 \pm 120$ & $2.81 \pm 0.23$ & $0.15 \pm 0.10$ & $1.47 \pm 0.13$ & -39.6 \\
\hline $\operatorname{Tr} 20$ & $12394514-6038258$ & $4930 \pm 120$ & $2.85 \pm 0.23$ & $0.12 \pm 0.10$ & $1.35 \pm 0.05$ & -40.7 \\
\hline $\operatorname{Tr} 20$ & $12394596-6038389$ & $4890 \pm 120$ & $2.75 \pm 0.24$ & $0.10 \pm 0.11$ & $1.42 \pm 0.06$ & -39.0 \\
\hline $\operatorname{Tr} 20$ & $12394688-6033540$ & $4960 \pm 120$ & $2.90 \pm 0.23$ & $0.14 \pm 0.09$ & $1.37 \pm 0.06$ & -40.2 \\
\hline $\operatorname{Tr} 20$ & $12394897-6033282$ & $4950 \pm 110$ & $2.81 \pm 0.22$ & $0.10 \pm 0.09$ & $1.50 \pm 0.09$ & -38.8 \\
\hline $\operatorname{Tr} 20$ & $12395554-6037268$ & $4900 \pm 110$ & $2.79 \pm 0.22$ & $0.13 \pm 0.10$ & $1.38 \pm 0.05$ & -39.8 \\
\hline $\operatorname{Tr} 20$ & $12395711-6039335$ & $4960 \pm 120$ & $2.83 \pm 0.23$ & $0.14 \pm 0.09$ & $1.44 \pm 0.06$ & -40.6 \\
\hline $\operatorname{Tr} 20$ & $12395973-6035072$ & $4880 \pm 110$ & $2.75 \pm 0.22$ & $0.10 \pm 0.10$ & $1.41 \pm 0.06$ & -41.1 \\
\hline $\operatorname{Tr} 20$ & $12400109-6031395$ & $4890 \pm 120$ & $2.85 \pm 0.22$ & $0.13 \pm 0.10$ & $1.39 \pm 0.04$ & -40.1 \\
\hline $\operatorname{Tr} 20$ & $12400116-6035218$ & $4890 \pm 120$ & $2.76 \pm 0.22$ & $0.10 \pm 0.10$ & $1.37 \pm 0.05$ & -39.3 \\
\hline $\operatorname{Tr} 20$ & $12400259-6039545$ & $4520 \pm 110$ & $2.20 \pm 0.22$ & $0.03 \pm 0.09$ & $1.47 \pm 0.15$ & -39.6 \\
\hline $\operatorname{Tr} 20$ & $12400754-6035445$ & $4430 \pm 110$ & $2.10 \pm 0.23$ & $0.06 \pm 0.10$ & $1.52 \pm 0.05$ & -39.5 \\
\hline $\operatorname{Tr} 20$ & $12402227-6037419$ & $4940 \pm 120$ & $2.77 \pm 0.23$ & $0.12 \pm 0.10$ & $1.50 \pm 0.10$ & -40.6 \\
\hline $\operatorname{Tr} 20$ & $12402478-6043103$ & $4590 \pm 120$ & $2.28 \pm 0.22$ & $0.03 \pm 0.11$ & $1.48 \pm 0.10$ & -39.1 \\
\hline $\operatorname{Tr} 23$ & $16003885-5334507$ & $4510 \pm 130$ & $2.42 \pm 0.23$ & $0.08 \pm 0.10$ & $1.49 \pm 0.29$ & -61.2 \\
\hline $\operatorname{Tr} 23$ & $16003935-5332367$ & $4800 \pm 120$ & $2.57 \pm 0.23$ & $0.14 \pm 0.10$ & $1.47 \pm 0.13$ & -60.9 \\
\hline $\operatorname{Tr} 23$ & $16004025-5329439$ & $4910 \pm 120$ & $2.77 \pm 0.23$ & $0.10 \pm 0.10$ & $1.51 \pm 0.14$ & -56.5 \\
\hline $\operatorname{Tr} 23$ & $16004312-5330509$ & $4910 \pm 120$ & $2.86 \pm 0.23$ & $0.11 \pm 0.10$ & $1.60 \pm 0.24$ & -61.8 \\
\hline $\operatorname{Tr} 23$ & $16005168-5332013$ & $4880 \pm 120$ & $2.60 \pm 0.23$ & $0.17 \pm 0.10$ & $1.71 \pm 0.16$ & -62.8 \\
\hline $\operatorname{Tr} 23$ & $16005220-5333362$ & $4920 \pm 120$ & $2.63 \pm 0.22$ & $0.18 \pm 0.10$ & $1.63 \pm 0.13$ & -62.4 \\
\hline $\operatorname{Tr} 23$ & $16005798-5331476$ & $4860 \pm 120$ & $2.69 \pm 0.23$ & $0.13 \pm 0.10$ & $1.45 \pm 0.07$ & -59.9 \\
\hline $\operatorname{Tr} 23$ & $16010025-5333101$ & $4880 \pm 120$ & $2.79 \pm 0.22$ & $0.10 \pm 0.10$ & $1.53 \pm 0.25$ & -60.2 \\
\hline $\operatorname{Tr} 23$ & $16010433-5332336$ & $4780 \pm 120$ & $2.48 \pm 0.24$ & $0.17 \pm 0.10$ & $1.68 \pm 0.18$ & -60.5 \\
\hline $\operatorname{Tr} 23$ & $16010639-5331056$ & $4850 \pm 120$ & $2.74 \pm 0.24$ & $0.14 \pm 0.11$ & $1.76 \pm 0.17$ & -61.9 \\
\hline $\operatorname{Tr} 23$ & $16010770-5329374$ & $4830 \pm 110$ & $2.63 \pm 0.23$ & $0.16 \pm 0.09$ & $1.47 \pm 0.12$ & -61.5 \\
\hline
\end{tabular}


L. Magrini et al.: Abundance ratios in the Galactic thin disc

Table A.2. Abundances in open cluster member stars.

\begin{tabular}{|c|c|c|c|c|c|c|c|c|c|c|}
\hline & & $\mathrm{O} / \mathrm{H}$ & $\mathrm{Mg} / \mathrm{H}$ & $\mathrm{Si} / \mathrm{H}$ & $\mathrm{Ca} / \mathrm{H}$ & $\mathrm{Ti} / \mathrm{H}$ & $\mathrm{Sc} / \mathrm{H}$ & $\mathrm{V} / \mathrm{H}$ & $\mathrm{Cr} / \mathrm{H}$ & $\mathrm{Ni} / \mathrm{H}$ \\
\hline $\mathrm{Br} 25$ & $6411039-1628006$ & & $7.41 \pm 0.12$ & $7.16 \pm 0.07$ & $5.97 \pm 0.09$ & $4.64 \pm 0.08$ & $2.94 \pm 0.07$ & $3.67 \pm 0.09$ & $5.28 \pm 0.12$ & $8.86 \pm 0.11$ \\
\hline & $6411363-1629172$ & $96 \pm 0.12$ & $44 \pm 0.12$ & $25 \pm 0.09$ & $.05 \pm 0.08$ & $4.66 \pm 0.10$ & $3.05 \pm 0.08$ & $3.60 \pm 0.10$ & $5.27 \pm 0.13$ & $5.93 \pm 0.13$ \\
\hline & & & & & & & & & & \\
\hline & & & & & & & & & & \\
\hline & $6411680-1630203$ & $87 \pm 0.12$ & & $7.26 \pm 0.07$ & $6.00 \pm 0.08$ & $4.69 \pm 0.08$ & $3.03 \pm 0.07$ & $3.70 \pm 0.09$ & $5.26 \pm 0.11$ & \\
\hline & & & & & & & & & & \\
\hline $\mathrm{Br} 44$ & & & & & & & & & & \\
\hline & $19170911+1933256$ & & & & & & & & & \\
\hline & & & & & & & & & & \\
\hline $\mathrm{Br} 44$ & & & & & & & & & & \\
\hline $\mathrm{Br} 81$ & $19013537-0028186$ & $8.92 \pm 0.33$ & & $7.70 \pm 0.07$ & & & $3.40 \pm 0.07$ & $4.54 \pm 0.08$ & $6.07 \pm 0.13$ & $6.68 \pm 0.11$ \\
\hline & & & & & & & & & & \\
\hline & & & & & & & & & & \\
\hline & & & & & & & & & & \\
\hline & & & & & & & & & & \\
\hline & & & & & & & & & & \\
\hline & & & & & & & & & & \\
\hline & & & & & & & & & & \\
\hline & & & & & & & & & & \\
\hline & & & & & & & & & & \\
\hline & & & & & & & & & & \\
\hline & & & & & & & & & & \\
\hline & & & & & & & & & & \\
\hline & & & & & & & & & & \\
\hline & & & & & & & & & & \\
\hline & & & & & & & & & & \\
\hline & & & & & & & & & & \\
\hline & & & & & & & & & & \\
\hline & & & & & & & & & & \\
\hline & & .08 & & & & & & & & \\
\hline & & & & & & & & & & \\
\hline & & & & & & & & & & \\
\hline & & & & & & & & & & \\
\hline & & & & & & & & & & \\
\hline & & & & & & & & & & \\
\hline & & & & & & & & & & \\
\hline & & & & & & & & & & \\
\hline & & & & & & & & & & \\
\hline & & & & & & & & & & \\
\hline & & & & & & & & & & \\
\hline & & & & & & & & & & \\
\hline & & & & & & & & & & \\
\hline & & & & & & & & & & \\
\hline & & & & & & & & & & \\
\hline & & & & & & & & & & \\
\hline & & & & & & & & & & \\
\hline & & & & & & & & & & \\
\hline & & & & & & & & & & \\
\hline & & & & & & & & & & \\
\hline & & & & & & & & & & \\
\hline & & & & & & & & & & \\
\hline & & & & & & & & & & \\
\hline & & & & & & & & & & \\
\hline & & & & & & & & & & \\
\hline & & & & & & & & & & \\
\hline & & & & & & & & & $8 \pm 0.10$ & \pm 0.11 \\
\hline & & & & & & & & & & \\
\hline & & & & & & & & & & \\
\hline & & & & & & & & & & \\
\hline & & & & & & & & & & \\
\hline & & & & & & & & & & \\
\hline & & & & & & & & & & \\
\hline & & & & & & & & & & \\
\hline & & & & & & & & & & \\
\hline & & & & & & & & & & \\
\hline & & & & & & & & & & \\
\hline & & & & & & & & & & \\
\hline & & & & & & & & & & \\
\hline & & & & & & & & & & \\
\hline NGC 6633 & $8283246+0644404$ & $8.99 \pm 0$. & $7.61 \pm 0$ & .07 & $6.36=$ & $4.87 \pm$ & $3.07 \pm 0$ & $3.87 \pm 0.09$ & $5.66 \pm 0.12$ & $6.08 \pm 0.10$ \\
\hline
\end{tabular}


Table A.2. continued.

\begin{tabular}{|c|c|c|c|c|c|c|c|c|c|c|}
\hline Cluster & CNAME & $\mathrm{O} / \mathrm{H}$ & $\mathrm{Mg} / \mathrm{H}$ & $\mathrm{Si} / \mathrm{H}$ & $\mathrm{Ca} / \mathrm{H}$ & $\mathrm{Ti} / \mathrm{H}$ & $\mathrm{Sc} / \mathrm{H}$ & $\mathrm{V} / \mathrm{H}$ & $\mathrm{Cr} / \mathrm{H}$ & $\mathrm{Ni} / \mathrm{H}$ \\
\hline NGC 6705 & $18502831-0615122$ & $8.78 \pm 0.11$ & $7.85 \pm 0.13$ & $7.61 \pm 0.07$ & $6.37 \pm 0.08$ & $4.92 \pm 0.08$ & $3.18 \pm 0.07$ & $4.00 \pm 0.09$ & $5.64 \pm 0.10$ & $6.38 \pm 0.12$ \\
\hline NGC 6705 & $18504563-0612038$ & $8.75 \pm 0.11$ & $7.87 \pm 0.12$ & $7.57 \pm 0.07$ & $6.37 \pm 0.09$ & $4.95 \pm 0.08$ & $3.13 \pm 0.08$ & $4.06 \pm 0.08$ & $5.62 \pm 0.10$ & $6.29 \pm 0.10$ \\
\hline NGC 6705 & $18505494-0616182$ & $.75 \pm 0.11$ & $7.85 \pm 0.12$ & $7.57 \pm 0.07$ & $6.37 \pm 0.09$ & $4.98 \pm 0.08$ & $3.20 \pm 0.07$ & $4.10 \pm 0.08$ & $5.65 \pm 0.11$ & $6.33 \pm 0.09$ \\
\hline NGC 6705 & $18510289-0615301$ & $.70 \pm 0.11$ & $7.77 \pm 0.12$ & $7.43 \pm 0.07$ & $6.37 \pm 0.09$ & $4.91 \pm 0.08$ & $3.15 \pm 0.07$ & $4.05 \pm 0.08$ & $5.65 \pm 0.10$ & $6.28 \pm 0.10$ \\
\hline NGC 6705 & $18510399-0620414$ & $8.76 \pm 0.11$ & $7.81 \pm 0.12$ & $7.60 \pm 0.07$ & $6.35 \pm 0.09$ & $4.86 \pm 0.08$ & $3.16 \pm 0.07$ & $3.97 \pm 0.09$ & $5.59 \pm 0.11$ & $6.34 \pm 0.10$ \\
\hline NGC 6705 & $18510662-0612442$ & $.82 \pm 0.11$ & $7.88 \pm 0.12$ & $7.59 \pm 0.07$ & $6.38 \pm 0.09$ & $5.01 \pm 0.08$ & $3.28 \pm 0.08$ & $4.11 \pm 0.09$ & $5.68 \pm 0.10$ & $6.36 \pm 0.10$ \\
\hline NGC 6705 & $18510786-0617119$ & $.75 \pm 0.11$ & $7.96 \pm 0.12$ & $7.63 \pm 0.07$ & $6.31 \pm 0.09$ & $4.93 \pm 0.08$ & $3.28 \pm 0.07$ & $4.05 \pm 0.09$ & $5.74 \pm 0.11$ & $6.37 \pm 0.10$ \\
\hline NGC 6705 & $18511116-0614340$ & $3.68 \pm 0.12$ & $7.84 \pm 0.13$ & $7.53 \pm 0.07$ & $6.26 \pm 0.09$ & $4.85 \pm 0.08$ & $3.02 \pm 0.07$ & $3.89 \pm 0.09$ & $5.60 \pm 0.11$ & $6.21 \pm 0.13$ \\
\hline NGC 6705 & $18511534-0618359$ & $8.75 \pm 0.12$ & $7.97 \pm 0.12$ & $7.65 \pm 0.07$ & $6.40 \pm 0.09$ & $5.03 \pm 0.08$ & $3.24 \pm 0.07$ & $4.14 \pm 0.09$ & $5.78 \pm 0.12$ & $6.45 \pm 0.11$ \\
\hline NGC 6705 & $18511571-0618146$ & $.75 \pm 0.11$ & $.81 \pm 0.12$ & $7.58 \pm 0.07$ & $6.29 \pm 0.08$ & $4.85 \pm 0.08$ & $3.22 \pm 0.07$ & $3.92 \pm 0.08$ & $5.56 \pm 0.11$ & $6.26 \pm 0.10$ \\
\hline NGC 6705 & $18512662-0614537$ & $8.68 \pm 0.12$ & $7.87 \pm 0.13$ & $7.65 \pm 0.07$ & $6.27 \pm 0.09$ & $4.84 \pm 0.07$ & $3.18 \pm 0.07$ & $3.78 \pm 0.09$ & $5.61 \pm 0.11$ & $6.30 \pm 0.13$ \\
\hline NGC 6705 & $18514034-0617128$ & $.72 \pm 0.11$ & $7.77 \pm 0.12$ & $7.53 \pm 0.07$ & $6.48 \pm 0.08$ & $4.96 \pm 0.08$ & $3.15 \pm 0.07$ & $4.05 \pm 0.08$ & $5.62 \pm 0.11$ & $6.33 \pm 0.10$ \\
\hline NGC 6705 & $18514130-0620125$ & $8.81 \pm 0.11$ & $7.82 \pm 0.12$ & $7.63 \pm 0.07$ & $6.36 \pm 0.09$ & $4.89 \pm 0.08$ & $3.25 \pm 0.07$ & $3.96 \pm 0.09$ & $5.66 \pm 0.10$ & $6.40 \pm 0.11$ \\
\hline NGC 6802 & & & & $7.53 \pm 0.07$ & & & $3.18 \pm 0.07$ & $3.99 \pm 0.09$ & & \\
\hline NGC 6802 & $19303085+2016274$ & $.71 \pm 0.12$ & $7.67 \pm 0.12$ & $7.53 \pm 0.07$ & $6.44 \pm 0.08$ & $4.99 \pm 0.08$ & $3.25 \pm 0.07$ & $4.03 \pm 0.09$ & $5.67 \pm 0.12$ & $6.30 \pm 0.11$ \\
\hline NGC 6802 & 19303 & & & $7.52 \pm 0.07$ & & & $3.14 \pm 0.07$ & & & \\
\hline NGC 6802 & $19303309+2015442$ & & $7.72 \pm 0.12$ & $7.55 \pm 0.07$ & $6.31 \pm 0.08$ & $4.89 \pm 0.08$ & $3.12 \pm 0.07$ & $3.95 \pm 0.09$ & & $6.24 \pm 0.09$ \\
\hline NGC 6802 & $19303611+2016329$ & $.80 \pm 0.12$ & $7.73 \pm 0.12$ & $7.53 \pm 0.07$ & $6.37 \pm 0.09$ & $4.91 \pm 0.08$ & $3.16 \pm 0.07$ & & & $6.25 \pm 0.10$ \\
\hline NGC 6802 & $884+2014005$ & & & $7.47 \pm 0.07$ & & $4.90 \pm 0.08$ & $3.23 \pm 0.07$ & & $5 \pm 0.11$ & \\
\hline NGC 6 & $19304170+2015224$ & & & $7.52 \pm 0.07$ & $6.39 \pm 0.08$ & $5.01 \pm 0.08$ & $3.33 \pm 0.07$ & $4.07 \pm 0.10$ & & \\
\hline Pis18 & $13365597-6205130$ & $8.74 \pm 0.11$ & $7.70 \pm 0.12$ & $7.54 \pm 0.07$ & $6.33 \pm 0.08$ & $4.89 \pm 0.08$ & $3.14 \pm 0.07$ & $4.00 \pm 0.09$ & $5.61 \pm 0.11$ & $6.22 \pm 0.11$ \\
\hline Pis 18 & $3-6206433$ & & & $7.54 \pm 0.07$ & & \pm 0.09 & $3.19 \pm 0.07$ & & 12 & $3 \pm 0.11$ \\
\hline Pis 18 & & & & $7.55 \pm 0.07$ & & $4.87 \pm 0.07$ & $3.21 \pm 0.07$ & $3.98 \pm 0.09$ & & \\
\hline $\operatorname{Tr} 20$ & $12383597-6045242$ & & & $7.55 \pm 0.07$ & & $4.96 \pm 0.08$ & $3.27 \pm 0.07$ & & & \\
\hline $\operatorname{Tr} 20$ & 1238 & & & & & & & & & \\
\hline $\operatorname{Tr} 20$ & & & & & & & & & & \\
\hline $\operatorname{Tr} 20$ & & & & $7.52 \pm 0.07$ & & & $3.29 \pm 0.07$ & & & \\
\hline $\operatorname{Tr} 20$ & 8402 & & & 0.07 & 0.09 & 0.08 & $3.14 \pm 0.07$ & 0.08 & & 0.11 \\
\hline $\operatorname{Tr} 20$ & 12391 & & & $=0.07$ & & 0.08 & 0.07 & & & \\
\hline $\operatorname{Tr} 20$ & & & & $=0.07$ & & & 0.07 & & & \\
\hline $\operatorname{Tr} 20$ & $12392636-6040217$ & & & 0.07 & & & $3.08 \pm 0.07$ & & & 0.09 \\
\hline $\operatorname{Tr} 20$ & & & & 0.07 & & $4.98 \pm 0.08$ & $3.23 \pm 0.07$ & $4.02 \pm 0.09$ & & \\
\hline $\operatorname{Tr} 20$ & & & & & & & & & & \\
\hline $\operatorname{Tr} 20$ & & & & & & & $3.15 \pm 0.07$ & & & \pm 0.09 \\
\hline $\operatorname{Tr} 20$ & & & & & & & & & & \\
\hline $\operatorname{Tr} 20$ & & & & 0.07 & & & 0.07 & & & \\
\hline $\operatorname{Tr} 20$ & 596-6038389 & & & $7.53 \pm 0.07$ & & $4.90 \pm 0.08$ & $3.16 \pm 0.07$ & $3.97 \pm 0.09$ & $5.62 \pm 0.12$ & $0 \pm 0.10$ \\
\hline $\operatorname{Tr} 20$ & 1239 & & & & & & & & & \\
\hline $\operatorname{Tr} 20$ & 282 & & 0.12 & 0.07 & 0.09 & 0.08 & 0.07 & & & 0.11 \\
\hline $\operatorname{Tr} 20$ & $54-6037268$ & & & $7.57 \pm 0.07$ & $6.42 \pm 0.08$ & $4.96 \pm 0.08$ & $3.27 \pm 0.07$ & & & $6.29 \pm 0.11$ \\
\hline $\operatorname{Tr} 20$ & $12395711-6039335$ & & & $7.60 \pm 0.07$ & & $4.99 \pm 0.08$ & $3.22 \pm 0.07$ & & $5.63 \pm 0.11$ & $6.31 \pm 0.11$ \\
\hline $\operatorname{Tr} 20$ & $73-6035072$ & & & 0.07 & & & & & & \\
\hline $\operatorname{Tr} 20$ & $09-6031395$ & & $7.72 \pm 0.12$ & $7.61 \pm 0.07$ & & & $3.28 \pm 0.07$ & $4.08 \pm 0.09$ & $5.71 \pm 0.12$ & $4 \pm 0.10$ \\
\hline $\operatorname{Tr} 20$ & $12400116-6035218$ & & $7.62 \pm 0.12$ & $7.54 \pm 0.07$ & & & $3.23 \pm 0.07$ & $4.01 \pm 0.09$ & $5.65 \pm 0.12$ & $8 \pm 0.10$ \\
\hline $\operatorname{Tr} 20$ & & & & & & & & & & \\
\hline & & & & & & & & & & \\
\hline $\operatorname{Tr} 20$ & $12402227-6037419$ & & $7.69 \pm 0.12$ & $7.32 \pm 0.07$ & $6.39 \pm 0.08$ & $4.93 \pm 0.08$ & $3.18 \pm 0.07$ & $4.02 \pm 0.09$ & $5.65 \pm 0.10$ & $6.31 \pm 0.09$ \\
\hline $\operatorname{Tr} 20$ & $12402478-6043103$ & & $7.66 \pm 0.12$ & $7.45 \pm 0.07$ & $6.35 \pm 0.08$ & $4.91 \pm 0.08$ & $3.20 \pm 0.07$ & $4.01 \pm 0.08$ & $5.61 \pm 0.11$ & $6.32 \pm 0.10$ \\
\hline $\operatorname{Tr} 23$ & $16003885-5334507$ & & & & & & & & & \\
\hline $\operatorname{Tr} 23$ & $16003935-5332367$ & $8.91 \pm 0.14$ & $7.87 \pm 0.12$ & $7.62 \pm 0.07$ & $6.43 \pm 0.09$ & $4.98 \pm 0.08$ & $3.26 \pm 0.07$ & $4.10 \pm 0.09$ & $5.73 \pm 0.13$ & $6.40 \pm 0.10$ \\
\hline $\operatorname{Tr} 23$ & $16004025-5329439$ & $8.78 \pm 0.11$ & $7.81 \pm 0.12$ & $7.64 \pm 0.07$ & $6.46 \pm 0.08$ & $4.96 \pm 0.08$ & $3.26 \pm 0.07$ & $4.11 \pm 0.09$ & $5.73 \pm 0.11$ & $6.31 \pm 0.11$ \\
\hline $\operatorname{Tr} 23$ & $16004312-5330509$ & & & & & & & & $5.76 \pm 0.11$ & \\
\hline $\operatorname{Tr} 23$ & $16005168-5332013$ & $8.95 \pm 0.25$ & & & & & & & & $6.38 \pm 0.09$ \\
\hline $\operatorname{Tr} 23$ & $16005220-5333362$ & $8.92 \pm 0.23$ & $7.88 \pm 0.12$ & $7.64 \pm 0.07$ & $6.43 \pm 0.08$ & $4.93 \pm 0.09$ & $3.28 \pm 0.07$ & $4.05 \pm 0.09$ & $5.66 \pm 0.12$ & $6.37 \pm 0.10$ \\
\hline $\operatorname{Tr} 23$ & $16005798-5331476$ & $8.81 \pm 0.12$ & $7.82 \pm 0.12$ & $7.66 \pm 0.07$ & $6.36 \pm 0.08$ & $4.92 \pm 0.09$ & $3.22 \pm 0.07$ & $4.05 \pm 0.09$ & $5.67 \pm 0.11$ & $6.30 \pm 0.11$ \\
\hline $\operatorname{Tr} 23$ & $16010025-5333101$ & $8.80 \pm 0.12$ & $7.67 \pm 0.12$ & $7.67 \pm 0.07$ & $6.42 \pm 0.08$ & $4.94 \pm 0.08$ & $3.27 \pm 0.06$ & & $5.73 \pm 0.12$ & $6.33 \pm 0.10$ \\
\hline $\operatorname{Tr} 23$ & $16010433-5332336$ & $8.80 \pm 0.11$ & $7.91 \pm 0.12$ & $7.60 \pm 0.07$ & $6.36 \pm 0.08$ & $4.95 \pm 0.08$ & $3.19 \pm 0.07$ & $4.07 \pm 0.08$ & $5.66 \pm 0.10$ & $6.33 \pm 0.10$ \\
\hline $\operatorname{Tr} 23$ & $16010639-5331056$ & $8.85 \pm 0.12$ & $7.78 \pm 0.12$ & $7.66 \pm 0.07$ & $6.33 \pm 0.08$ & $4.96 \pm 0.08$ & $3.28 \pm 0.07$ & $4.04 \pm 0.08$ & $5.66 \pm 0.11$ & $6.31 \pm 0.10$ \\
\hline $\operatorname{Tr} 23$ & $16010770-5329374$ & $8.79 \pm 0.33$ & $7.86 \pm 0.12$ & $7.71 \pm 0.07$ & $6.48 \pm 0.08$ & $5.06 \pm 0.08$ & $3.31 \pm 0.06$ & $4.15 \pm 0.09$ & $5.78 \pm 0.11$ & $6.44 \pm 0.10$ \\
\hline
\end{tabular}


L. Magrini et al.: Abundance ratios in the Galactic thin disc

Table A.3. Stellar parameters, ages, distances and heights of Milky Way field stars.

\begin{tabular}{|c|c|c|c|c|c|c|c|}
\hline CNAME & $\begin{array}{l}T_{\text {eff }} \\
(\mathrm{K})\end{array}$ & $\log g$ & $\begin{array}{c}{[\mathrm{Fe} / \mathrm{H}]} \\
(\mathrm{dex})\end{array}$ & $\begin{array}{c}\xi \\
\left(\mathrm{km} \mathrm{s}^{-1}\right) \\
\end{array}$ & $\begin{array}{l}\text { Age } \\
(\mathrm{Gyr})\end{array}$ & $\begin{array}{c}R \\
(\mathrm{kpc})\end{array}$ & $\begin{array}{c}Z \\
(\mathrm{kpc})\end{array}$ \\
\hline \multicolumn{8}{|l|}{ Solar neighbourhood } \\
\hline $07033617-4229583$ & $6460 \pm 170$ & $4.25 \pm 0.30$ & $0.07 \pm 0.11$ & $1.93 \pm 0.02$ & $1.6 \pm 0.9$ & $8.17 \pm 0.04$ & $-0.15 \pm 0.03$ \\
\hline $07040713-4235207$ & $6400 \pm 120$ & & $-0.30 \pm 0.09$ & $1.64 \pm 0.08$ & & $8.19 \pm 0.06$ & $-0.17 \pm 0.05$ \\
\hline $07202470-0022310$ & $6400 \pm 120$ & $4.00 \pm 0.23$ & $-0.32 \pm 0.10$ & $1.25 \pm 0.11$ & $3.9 \pm 1.5$ & $9.50 \pm 0.40$ & $0.20 \pm 0.05$ \\
\hline $07203700-0031573$ & $5800 \pm 110$ & $4.40 \pm 0.24$ & $0.01 \pm 0.09$ & $1.26 \pm 0.05$ & $4.8 \pm 2.9$ & $8.59 \pm 0.09$ & $0.08 \pm 0.01$ \\
\hline $07451083-4229336$ & $6260 \pm 340$ & $3.80 \pm 0.72$ & $-0.49 \pm 0.15$ & $1.64 \pm 0.50$ & $4.5 \pm 2.9$ & $8.35 \pm 0.14$ & $-0.18 \pm 0.06$ \\
\hline $07553858-0901268$ & $6360 \pm 120$ & $4.00 \pm 0.23$ & $0.07 \pm 0.09$ & $1.58 \pm 0.07$ & & $8.33 \pm 0.08$ & $0.09 \pm 0.02$ \\
\hline 07562014-0905179 & $6600 \pm 160$ & $3.90 \pm 0.25$ & $-0.27 \pm 0.11$ & $1.82 \pm 0.06$ & $2.8 \pm 1.1$ & $.60 \pm 0.18$ & $0.15 \pm 0.04$ \\
\hline $08002562-0023500$ & $6750 \pm 170$ & $4.20 \pm 0.28$ & $-0.63 \pm 0.10$ & $1.40 \pm 0.03$ & $2.9 \pm 1.6$ & $52 \pm 0.10$ & $0.18 \pm 0.06$ \\
\hline & $6050 \pm 110$ & $4.00 \pm 0.23$ & $0.04 \pm 0.09$ & $1.32 \pm 0.07$ & $4.1 \pm 1.7$ & $.44 \pm 0.12$ & $0.16 \pm 0.04$ \\
\hline $08403011-1353373$ & $5960 \pm 110$ & & & & & & $0.19 \pm 0.06$ \\
\hline 09592773-4054107 & $6290 \pm 120$ & $4.20 \pm 0.23$ & $0.02 \pm 0.09$ & $1.40 \pm 0.04$ & $2.1 \pm 1.1$ & $01 \pm 0.00$ & $0.18 \pm 0.02$ \\
\hline & $5850 \pm 130$ & & & & & & \\
\hline $10144572-4050005$ & $6000 \pm 110$ & $4.10 \pm 0.22$ & & & & $98 \pm 0.01$ & $0.11 \pm 0.04$ \\
\hline $2650-4104417$ & $6100 \pm 120$ & & & & & & $0.19 \pm 0.03$ \\
\hline & $6100 \pm 120$ & & & & & & $0.16 \pm 0.06$ \\
\hline $13592093-4058230$ & $5830 \pm 140$ & & & & & & $0.11 \pm 0.01$ \\
\hline 145 & $6200 \pm 120$ & & & & & & \\
\hline 145 & $400 \pm 120$ & 4.1 & -0.1 & & & & \\
\hline & $60 \pm 120$ & & & & & & \\
\hline & & & & & & & \\
\hline & & & & & & & \\
\hline & & & & & & & \\
\hline & & & & & & & \\
\hline $15234823-4219531$ & $6150 \pm 110$ & 4.31 & 0.10 & $1.35 \pm$ & $.4 \pm 1.4$ & \pm 0.12 & \pm 0.03 \\
\hline $4475-4412297$ & & & & & & & \\
\hline & & & & & & & \\
\hline $15540366-40$ & $6000 \pm 110$ & & 0.09 & & & 12 & 0.02 \\
\hline & & & & & & & \\
\hline & & & & & & & \\
\hline & & & & & & & \\
\hline & & & & & & & \\
\hline & & & & & & & $-0.10 \pm 0.02$ \\
\hline $18130118-4224479$ & $5900 \pm 110$ & & & & & & $-0.17 \pm 0.04$ \\
\hline \multicolumn{8}{|l|}{ Inner disc } \\
\hline & & & & & & & \\
\hline & & & & & & & \\
\hline & & & & & & & 0.05 \\
\hline & & & & & & & $-0.18 \pm 0.05$ \\
\hline & & & & & & & \\
\hline & & & & & & & $=0.05$ \\
\hline $75-2959121$ & $4600 \pm 125$ & $2.50 \pm 0.23$ & $-0.00 \pm 0.10$ & & & & $-0.18 \pm 0.05$ \\
\hline & & & & & & & $-0.17 \pm 0.05$ \\
\hline & $4600 \pm 120$ & & & & & & $=0.05$ \\
\hline & $5000 \pm 110$ & & & & & & $-0.17 \pm 0.07$ \\
\hline & & & & & & & \\
\hline & $400 \pm 135$ & & & & $4.8 \pm 3.5$ & & 0.05 \\
\hline & & & & & & & \pm 0.07 \\
\hline & & & & & & & \pm 0.05 \\
\hline $0712-3154573$ & $4700 \pm 130$ & $2.44 \pm 0$. & $-0.05 \pm 0.10$ & $1.45 \pm 0.07$ & $3.3 \pm 3.2$ & $6.50 \pm 0.50$ & $-0.16 \pm 0.05$ \\
\hline & & & & & & & $-0.16 \pm 0.05$ \\
\hline & & & & & & & $-0.15 \pm 0.07$ \\
\hline $2348-2441039$ & $4900 \pm 120$ & $2.80 \pm 0$. & $0.09 \pm 0.10$ & $1.39 \pm 0$. & $1.0 \pm 1.0$ & $6.35 \pm 0.40$ & $-0.15 \pm 0.03$ \\
\hline & & & & & & & $-0.15 \pm 0.04$ \\
\hline & & & & & & & $-0.15 \pm 0.04$ \\
\hline $18094596-3154021$ & $5100 \pm 120$ & $3.06 \pm 0$. & $-0.10 \pm 0.10$ & $1.21 \pm 0.05$ & $1.2 \pm 1.4$ & $6.55 \pm 0.60$ & $-0.15 \pm 0.06$ \\
\hline & & & & & & & \\
\hline $3242848-2445068$ & $4700 \pm 125$ & $2.73 \pm 0$ & & $1.39 \pm 0$ & & $6.45 \pm 0.40$ & $-0.15 \pm 0.04$ \\
\hline $8104764-3148047$ & $4700 \pm 120$ & $3.11 \pm 0$ & $0.03 \pm 0.09$ & $1.07 \pm 0$ & $4.8 \pm$ & $6.60 \pm 0.70$ & $-0.14 \pm 0.07$ \\
\hline & & & & & & & \\
\hline $18242463-2434518$ & $4700 \pm 120$ & $2.55 \pm 0.22$ & $0.11 \pm 0.09$ & $1.38 \pm 0.04$ & $2.1 \pm 2.3$ & $6.60 \pm 0.40$ & $-0.13 \pm 0.03$ \\
\hline
\end{tabular}


A\&A 603, A2 (2017)

Table A.4. Elemental abundances in the $12+\log (\mathrm{X} / \mathrm{H})$ form of Milky Way field stars.

\begin{tabular}{|c|c|c|c|c|c|c|c|c|c|}
\hline CNAME & $\mathrm{O} / \mathrm{H}$ & $\mathrm{Mg} / \mathrm{H}$ & $\mathrm{Si} / \mathrm{H}$ & $\mathrm{Ca} / \mathrm{H}$ & $\mathrm{Ti} / \mathrm{H}$ & $\mathrm{Sc} / \mathrm{H}$ & $\mathrm{Cr} / \mathrm{H}$ & $\mathrm{V} / \mathrm{H}$ & $\mathrm{Ni} / \mathrm{H}$ \\
\hline \multicolumn{10}{|l|}{ Solar neighbourhood } \\
\hline $07033617-4229583$ & $.76 \pm 0.22$ & $7.48 \pm 0.12$ & $7.64 \pm 0.07$ & $6.52 \pm 0.10$ & $5.24 \pm 0.11$ & $3.55 \pm 0.08$ & $4.21 \pm 0.11$ & $5.83 \pm 0.12$ & $6.52 \pm 0.10$ \\
\hline $07040713-4235207$ & $8.46 \pm 0.13$ & $7.47 \pm 0.12$ & $7.14 \pm 0.07$ & $5.12 \pm 0.09$ & $4.73 \pm 0.10$ & $2.82 \pm 0.07$ & $3.66 \pm 0.13$ & $5.30 \pm 0.13$ & $5.90 \pm 0.10$ \\
\hline $07203700-0031573$ & $8.78 \pm 0.14$ & $7.64 \pm 0.12$ & $7.31 \pm 0.07$ & $6.34 \pm 0.08$ & $4.86 \pm 0.09$ & $3.13 \pm 0.07$ & $3.93 \pm 0.10$ & $5.57 \pm 0.11$ & $6.17 \pm 0.10$ \\
\hline $07451083-4229336$ & $9.44 \pm 0.12$ & $6.93 \pm 0.13$ & $6.68 \pm 0.10$ & $6.08 \pm 0.10$ & $4.84 \pm 0.14$ & $2.80 \pm 0.18$ & $4.55 \pm 0.18$ & $5.20 \pm 0.15$ & $6.33 \pm 0.17$ \\
\hline $07553858-0901268$ & $9.52 \pm 0.12$ & $7.57 \pm 0.12$ & $7.41 \pm 0.07$ & $6.36 \pm 0.09$ & $4.96 \pm 0.09$ & $3.12 \pm 0.07$ & $4.12 \pm 0.10$ & $5.60 \pm 0.12$ & $6.14 \pm 0.10$ \\
\hline $07562014-0905179$ & $8.32 \pm 0.39$ & $7.62 \pm 0.12$ & $7.37 \pm 0.07$ & $6.25 \pm 0.10$ & $4.97 \pm 0.11$ & $3.06 \pm 0.08$ & $4.01 \pm 0.14$ & $5.58 \pm 0.13$ & $6.11 \pm 0.12$ \\
\hline $08002562-0023500$ & $8.52 \pm 0.17$ & $7.11 \pm 0.14$ & $7.03 \pm 0.08$ & $5.98 \pm 0.09$ & $4.79 \pm 0.11$ & $2.73 \pm 0.08$ & $3.79 \pm 0.18$ & $5.28 \pm 0.14$ & $5.89 \pm 0.12$ \\
\hline $08195062-1405598$ & $8.72 \pm 0.11$ & $7.68 \pm 0.12$ & $7.41 \pm 0.07$ & $6.28 \pm 0.08$ & $4.84 \pm 0.08$ & $3.12 \pm 0.07$ & $3.90 \pm 0.11$ & $5.46 \pm 0.11$ & $6.11 \pm 0.09$ \\
\hline $08403011-1353373$ & $8.76 \pm 0.12$ & $7.51 \pm 0.12$ & $7.44 \pm 0.07$ & $6.25 \pm 0.08$ & $4.78 \pm 0.09$ & $3.09 \pm 0.07$ & $3.85 \pm 0.10$ & $5.50 \pm 0.12$ & $6.10 \pm 0.10$ \\
\hline $09592773-4054107$ & $8.86 \pm 0.11$ & $7.49 \pm 0.12$ & $7.46 \pm 0.07$ & $6.29 \pm 0.09$ & $4.84 \pm 0.10$ & $3.13 \pm 0.07$ & $3.86 \pm 0.13$ & $5.61 \pm 0.11$ & $6.16 \pm 0.10$ \\
\hline $10000042-4050203$ & $8.21 \pm 0.33$ & $7.95 \pm 0.12$ & $7.65 \pm 0.07$ & $6.51 \pm 0.08$ & $5.21 \pm 0.09$ & $3.52 \pm 0.07$ & $4.30 \pm 0.09$ & $5.88 \pm 0.12$ & $6.55 \pm 0.11$ \\
\hline $10144572-4050005$ & $8.53 \pm 0.12$ & $7.63 \pm 0.12$ & $7.41 \pm 0.07$ & $6.26 \pm 0.08$ & $4.82 \pm 0.09$ & $3.12 \pm 0.07$ & $3.87 \pm 0.10$ & $5.54 \pm 0.12$ & $6.12 \pm 0.10$ \\
\hline $10392650-4104417$ & $8.32 \pm 0.32$ & $7.50 \pm 0.12$ & $7.39 \pm 0.07$ & $6.25 \pm 0.09$ & $4.82 \pm 0.09$ & $3.06 \pm 0.07$ & $3.84 \pm 0.11$ & $5.47 \pm 0.13$ & $6.04 \pm 0.11$ \\
\hline $11004237-4053156$ & $8.90 \pm 0.11$ & $7.81 \pm 0.12$ & $7.63 \pm 0.07$ & $6.48 \pm 0.09$ & $5.09 \pm 0.10$ & $3.37 \pm 0.07$ & $4.05 \pm 0.11$ & $5.75 \pm 0.11$ & $6.38 \pm 0.10$ \\
\hline $13592093-4058230$ & $8.92 \pm 0.12$ & $7.74 \pm 0.12$ & $7.69 \pm 0.07$ & $6.51 \pm 0.09$ & $5.21 \pm 0.09$ & $3.67 \pm 0.08$ & $4.33 \pm 0.10$ & $5.93 \pm 0.11$ & $6.58 \pm 0.11$ \\
\hline $14581143-4056574$ & $9.30 \pm 0.12$ & $7.39 \pm 0.12$ & $7.38 \pm 0.07$ & $6.31 \pm 0.08$ & $4.88 \pm 0.11$ & $3.03 \pm 0.08$ & & & \\
\hline $14582064-4104055$ & $9.30 \pm 0.12$ & $7.82 \pm 0.12$ & $7.70 \pm 0.07$ & $6.52 \pm 0.08$ & $5.12 \pm 0.08$ & $3.48 \pm 0.07$ & $4.19 \pm 0.10$ & $5.86 \pm 0.12$ & $6.52 \pm 0.10$ \\
\hline $15104258-4058579$ & $9.70 \pm 0.12$ & $8.01 \pm 0.13$ & $7.85 \pm 0.07$ & $6.70 \pm 0.08$ & $5.44 \pm 0.08$ & $3.71 \pm 0.07$ & $4.50 \pm 0.09$ & $6.09 \pm 0.11$ & $6.77 \pm 0.10$ \\
\hline $15170683-4131223$ & $29 \pm 0.12$ & $7.57 \pm 0.12$ & $7.46 \pm 0$ & & $4.88 \pm 0.09$ & $3.17 \pm 0$ & $3.87 \pm 0.09$ & 5.5 & $6.19 \pm 0.10$ \\
\hline $15174505-4126151$ & & $7.58 \pm 0.12$ & $7.46 \pm 0$ & $6.39 \pm 0.09$ & $4.93 \pm 0.09$ & $3.23 \pm 0$ & & & \\
\hline $15230726-4221416$ & $8.84 \pm 0.12$ & $7.80 \pm 0.12$ & $7.62 \pm 0.07$ & $6.47 \pm 0.08$ & $5.04 \pm 0.08$ & $3.40 \pm 0.07$ & $4.11 \pm 0.10$ & & $6.41 \pm 0.10$ \\
\hline $15234823-4219531$ & $.84 \pm 0.13$ & $7.73 \pm 0.12$ & $7.55 \pm 0.07$ & $6.42 \pm 0.08$ & $5.01 \pm 0.09$ & $3.30 \pm 0.07$ & $4.03 \pm 0.11$ & $5.64 \pm 0.12$ & $6.26 \pm 0.10$ \\
\hline & & & & & & & & & \\
\hline $15421514-4419378$ & $8.99 \pm 0.12$ & & & & $5.08 \pm 0.09$ & & & & \\
\hline $15540366-4052141$ & $8.66 \pm 0.12$ & $7.71 \pm 0.12$ & $7.52 \pm 0.07$ & $6.41 \pm 0.08$ & $4.95 \pm 0.09$ & $3.28 \pm 0.07$ & $3.94 \pm 0.10$ & $5.63 \pm 0.12$ & $6.28 \pm 0.10$ \\
\hline $17332693-4253210$ & $.27 \pm 0.12$ & $7.47 \pm 0.12$ & $7.52 \pm 0.07$ & $6.58 \pm 0.08$ & $5.16 \pm 0.10$ & $3.28 \pm 0.08$ & $4.30 \pm 0.11$ & $5.82 \pm 0$ & $6.30 \pm 0.10$ \\
\hline $17335392-4255153$ & & $7.69 \pm 0.12$ & & & $5.24 \pm 0.09$ & $3.39 \pm 0$ & & & \\
\hline $17335667-4302456$ & $9.65 \pm 0.12$ & $7.88 \pm 0.12$ & $7.53 \pm 0.07$ & $6.50 \pm 0.08$ & $5.32 \pm 0.11$ & $3.65 \pm 0.07$ & & & \\
\hline $17345383-4300502$ & $8.85 \pm 0.11$ & $7.74 \pm 0.12$ & $7.48 \pm 0.07$ & $6.40 \pm 0.09$ & $4.96 \pm 0.08$ & $3.34 \pm 0.07$ & $4.07 \pm 0.09$ & $5.71 \pm 0.11$ & $6.33 \pm 0.10$ \\
\hline $17583518-4218175$ & & $7.93 \pm 0.12$ & $7.79 \pm 0.07$ & & $5.15 \pm 0.09$ & $3.58 \pm 0$ & $4.25 \pm 0.09$ & $5.92 \pm 0$ & $6.62 \pm 0.10$ \\
\hline $18130118-4224479$ & & $7.83=$ & $7.64 \pm 0.07$ & $46 \pm 0.08$ & $5.05 \pm 0.10$ & $3.35 \pm 0.07$ & & & $6.39 \pm$ \\
\hline \multicolumn{10}{|l|}{ Inner disc } \\
\hline $40121-3003154$ & $8.91 \pm 0.12$ & $7.70 \pm 0.12$ & $7.48 \pm 0.07$ & $6.26 \pm 0.08$ & $4.85 \pm 0.08$ & $3.26 \pm 0.07$ & $4.00 \pm 0.08$ & $5.53 \pm 0.12$ & $6.31 \pm 0.10$ \\
\hline $381-2441158$ & $8.92 \pm 0.11$ & $7.85 \pm 0.12$ & $7.64 \pm 0.07$ & $39 \pm 0.09$ & $4.99 \pm 0.08$ & $3.28 \pm 0.07$ & \pm 0.09 & $5.68 \pm 0.11$ & \pm 0.11 \\
\hline $18231983-3407192$ & $8.83 \pm 0.12$ & $7.87 \pm 0.13$ & $7.68 \pm 0.07$ & $6.36 \pm 0.08$ & $4.88 \pm 0.08$ & $3.23 \pm 0.07$ & $4.04 \pm 0.08$ & $5.65 \pm 0.11$ & $6.43 \pm 0.10$ \\
\hline $18035275-2959121$ & $8.74 \pm 0.30$ & $7.74 \pm 0.12$ & $7.42 \pm 0.07$ & $6.21 \pm 0.08$ & $4.87 \pm 0.08$ & $3.21 \pm 0.08$ & $3.95 \pm 0.08$ & $5.52 \pm 0.10$ & $6.28 \pm 0.11$ \\
\hline $18253272-2510464$ & $8.85 \pm 0.11$ & $7.75 \pm 0.12$ & $7.53 \pm 0.07$ & $6.31 \pm 0.08$ & $4.91 \pm 0.08$ & $3.24 \pm 0.07$ & $4.00 \pm 0.09$ & $5.55 \pm 0.11$ & $6.30 \pm 0.11$ \\
\hline $18230343-3414129$ & $8.81 \pm 0.12$ & $7.73 \pm 0.12$ & $7.53 \pm 0.07$ & $6.33 \pm 0.08$ & $4.89 \pm 0.08$ & $3.20 \pm 0.07$ & $4.04 \pm 0.08$ & $5.55 \pm 0.10$ & $6.27 \pm 0.10$ \\
\hline $18224056-3401097$ & $8.85 \pm 0.12$ & $7.71 \pm 0.12$ & $7.54 \pm 0.07$ & $6.44 \pm 0.08$ & $4.96 \pm 0.08$ & $3.25 \pm 0.07$ & $4.03 \pm 0.09$ & $5.63 \pm 0.10$ & $6.30 \pm 0.10$ \\
\hline $18185651-3252428$ & & & & & & & & & $6.57 \pm 0.11$ \\
\hline & & & & & & & & & \\
\hline $18253617-2518331$ & $9.03 \pm 0.12$ & $7.86 \pm 0.12$ & $7.61 \pm 0.07$ & $6.43 \pm 0.08$ & $5.05 \pm 0.08$ & $3.40 \pm 0.06$ & $4.17 \pm 0.09$ & $5.70 \pm 0.11$ & $6.47 \pm 0.09$ \\
\hline $18035677-3017252$ & $9.05 \pm 0.12$ & $7.76 \pm 0.12$ & $7.55 \pm 0.07$ & $6.33 \pm 0.08$ & $4.96 \pm 0.07$ & $3.13 \pm 0.07$ & $4.06 \pm 0.08$ & $5.56 \pm 0.11$ & $6.31 \pm 0.10$ \\
\hline $18100712-3154573$ & & $7.52 \pm 0.12$ & & & & & & $5.47 \pm 0.11$ & $6.19 \pm 0.10$ \\
\hline & & & & & & & & & \\
\hline $18225163-3408040$ & $8.80 \pm 0.12$ & $7.72 \pm 0.12$ & $7.48 \pm 0.07$ & $6.28 \pm 0.08$ & $4.88 \pm 0.08$ & $3.21 \pm 0.07$ & $4.00 \pm 0.08$ & $5.53 \pm 0.11$ & $6.24 \pm 0.10$ \\
\hline $18242348-2441039$ & $8.75 \pm 0.12$ & $7.72 \pm 0.12$ & $7.58 \pm 0.07$ & $6.42 \pm 0.08$ & $4.94 \pm 0.08$ & $3.26 \pm 0.07$ & $4.10 \pm 0.09$ & $5.69 \pm 0.11$ & $6.31 \pm 0.11$ \\
\hline $18031173-3001517$ & $8.89 \pm 0.12$ & $7.89 \pm 0.12$ & $7.57 \pm 0.07$ & $6.54 \pm 0.08$ & $5.06 \pm 0.08$ & $3.41 \pm 0.08$ & $4.19 \pm 0.08$ & $5.77 \pm 0.12$ & $6.55 \pm 0.09$ \\
\hline $18105528-3148003$ & $8.66 \pm 0.11$ & $7.51 \pm 0.12$ & $7.31 \pm 0.07$ & $6.13 \pm 0.08$ & $4.70 \pm 0.08$ & $3.04 \pm 0.07$ & $3.72 \pm 0.09$ & $5.34 \pm 0.11$ & $6.00 \pm 0.10$ \\
\hline $18094596-3154021$ & $8.71 \pm 0.12$ & $7.56 \pm 0.12$ & $7.34 \pm 0.07$ & $6.20 \pm 0.08$ & $4.74 \pm 0.08$ & $3.13 \pm 0.07$ & $3.74 \pm 0.09$ & $5.43 \pm 0.12$ & $6.05 \pm 0.10$ \\
\hline $18030864-3017339$ & $8.76 \pm 0.11$ & $7.56 \pm 0.12$ & $7.39 \pm 0.07$ & $6.18 \pm 0.09$ & $4.76 \pm 0.08$ & $3.10 \pm 0.07$ & $3.78 \pm 0.08$ & $5.33 \pm 0.12$ & $6.10 \pm 0.10$ \\
\hline $18242848-2445068$ & $9.16 \pm 0.12$ & $7.84 \pm 0.12$ & $7.67 \pm 0.07$ & $6.45 \pm 0.08$ & $5.02 \pm 0.08$ & $3.32 \pm 0.07$ & $4.13 \pm 0.09$ & $5.77 \pm 0.11$ & $6.46 \pm 0.10$ \\
\hline $18104764-3148047$ & $8.82 \pm 0.12$ & $7.66 \pm 0.12$ & $7.5 \pm 0.07$ & $6.35 \pm 0.08$ & $4.93 \pm 0.08$ & $3.27 \pm 0.07$ & $4.05 \pm 0.09$ & $5.58 \pm 0.11$ & $6.28 \pm 0.10$ \\
\hline $17553060-3742441$ & $8.68 \pm 0.11$ & $7.53 \pm 0.12$ & $7.35 \pm 0.07$ & $6.2 \pm 0.08$ & $4.74 \pm 0.08$ & $3.07 \pm 0.07$ & $3.77 \pm 0.08$ & $5.39 \pm 0.11$ & $6.00 \pm 0.10$ \\
\hline $18242463-2434518$ & $8.84 \pm 0.12$ & $7.77 \pm 0.12$ & $7.61 \pm 0.07$ & $6.38 \pm 0.09$ & $4.93 \pm 0.08$ & $3.26 \pm 0.07$ & $4.09 \pm 0.08$ & $5.65 \pm 0.11$ & $6.37 \pm 0.10$ \\
\hline
\end{tabular}

